The AstronOMical Journal, 116:2341-2362, 1998 November

(C) 1998. The American Astronomical Society. All rights reserved. Printed in U.S.A.

\title{
THE RECENT STAR FORMATION IN SEXTANS A
}

\author{
SCHUYLER D. VAN DYK ${ }^{1}$ \\ Infrared Processing and Analysis Center, California Institute of Technology, Mail Code 100-22, Pasadena, CA 91125; vandyk@ipac.caltech.edu \\ DANIEL PUChE ${ }^{1}$ \\ Tellabs Transport Group, Inc., 3403 Griffith Street, Ville St. Laurent, Montreal, Quebec H4T 1W5, Canada; Daniel.Puche@tellabs.com \\ AND \\ TONY WONG ${ }^{1}$ \\ Department of Astronomy, University of California at Berkeley, 601 Cambell Hall, Berkeley, CA 94720-3411; twong@astro.berkeley.edu \\ Received 5 February 1998; revised 1998 July 8
}

\begin{abstract}
We investigate the relationship between the spatial distributions of stellar populations and of neutral and ionized gas in the Local Group dwarf irregular galaxy Sextans A. This galaxy is currently experiencing a burst of localized star formation, the trigger of which is unknown. We have resolved various populations of stars via deep $U B V(R I)_{\mathrm{C}}$ imaging over an area with diameter $\sim 5.3$. We have compared our photometry with theoretical isochrones appropriate for Sextans A, in order to determine the ages of these populations. We have mapped out the history of star formation, most accurately for times $\lesssim 100$ Myr. We find that star formation in Sextans A is correlated both in time and space, especially for the most recent $(\lesssim 12 \mathrm{Myr}$ ) times. The youngest stars in the galaxy are forming primarily along the inner edge of the large $\mathrm{H}$ I shell. Somewhat older populations, $\lesssim 50 \mathrm{Myr}$, are found inward of the youngest stars. Progressively older star formation, from $\sim 50-100 \mathrm{Myr}$, appears to have some spatially coherent structure and is more centrally concentrated. The oldest stars we can accurately sample appear to have approximately a uniform spatial distribution, which extends beyond a surface brightness of $\mu_{B} \simeq 25.9$ mag $\operatorname{arcsec}^{-2}$ (or, a radius $r \simeq 2.3$ ). Although other processes are also possible, our data provide support for a mechanism of supernova-driven expansion of the neutral gas, resulting in cold-gas pileup and compression along the $\mathrm{H}$ i shell and sequential star formation in recent times.
\end{abstract}

Key words: galaxies: dwarf - galaxies: evolution — galaxies: individual (Sextans A, DDO 75) galaxies: photometry - galaxies: stellar content — Local Group

\section{INTRODUCTION}

Dwarf galaxies, both irregular and spheroidal, are the most numerous galaxies in the universe. Dwarf irregular galaxies play a key role in our understanding of galactic evolution, especially with the discoveries of faint, blue galaxies in high-redshift imaging surveys such as the Hubble Deep Field. Since dwarf irregular galaxies are simpler, less evolved systems, it should be possible to observe short-lived evolutionary properties in nearby systems, which would be more easily destroyed in more massive, complex systems, such as grand-design spiral galaxies. Knowledge of the star formation history of dwarf irregular galaxies is of particular importance; Hunter (1997) recently presented a comprehensive discussion of the topic.

Dwarf irregular galaxies show a larger range in star formation rates than do spiral galaxies. Studies of the stellar populations in dwarf irregular galaxies (e.g., Tosi et al. 1991; Greggio et al. 1993; Marconi et al. 1995) show that most of these galaxies evolve with approximately constant star formation rates, either high or low, interrupted by variations of factors 2-3 in these rates over the past few gigayears (Hunter 1997). The question, then, is what governs and regulates star formation in dwarf irregular galaxies. A vital clue to answering this question can be found in the relationship between the structure and kinematics of the

\footnotetext{
${ }^{1}$ Visiting Astronomer, Kitt Peak National Observatory, National Optical Astronomy Observatories, which is operated by the Association of Universities for Research in Astronomy (AURA), Inc., under contract with the National Science Foundation.
}

gas and the history of star formation. The gas can be traced and analyzed by mapping the neutral, molecular, and ionized components, and the star formation history can best be traced by the distributions of resolved stars of various ages.

A program to study the interstellar medium (ISM) in dwarf irregular galaxies was undertaken by D. Puche, D. Westpfahl, and their collaborators, with the goal of determining whether the neutral component of the ISM is distributed differently in low-mass systems than in granddesign spiral galaxies. Puche et al. (1992), Westpfahl \& Puche (1994), and Puche \& Westpfahl (1994) found that, based on their high-resolution $\mathrm{H}$ I data for a number of dwarf galaxies, the neutral gas component shows an unprecedented amount of structure in the form of shells or bubbles (Heiles 1979, 1984; Tenorio-Tagle \& Bodenheimer 1988). Low-rotation velocities for dwarf galaxies means less shear, and thus, longer lifetimes for these large-scale shells. Furthermore, the gas scale-height in dwarf galaxies must be much larger than in high-mass spiral galaxies, resulting in a lower gas density and a higher efficiency for making large holes (Heiles 1979). Also important, of course, is the absence of spiral density waves (see Hunter, Elmegreen, \& Baker 1998).

Puche et al. (1992), Puche \& Westpfahl (1994), and Westpfahl \& Puche (1994) found that these structures are expanding and argued that they surround hot, low-density bubbles formed as the result of the combined effects of photoionization, stellar winds, and multiple supernovae from sequential formation of massive stars (Cash et al. 1980). Supernovae would likely dominate all other sources 
of energy input, although winds from massive stars also substantially contribute (e.g., Leitherer, Robert, \& Drissen 1992). The models of Chevalier (1974) and Koo \& McKee (1992a, 1992b) predict that about 100 supernovae exploding in preexisting wind-blown bubbles would provide about $10^{53}$ ergs to the ISM and can produce shells with radii of hundreds of parsecs, expansion velocities of $\sim 10-20 \mathrm{~km}$ $\mathrm{s}^{-1}$, and lifetimes of $\sim 10^{8} \mathrm{yr}$, consistent with those observed.

If supernovae are producing expanding bubbles, Puche $\&$ Westpfahl have argued, the current star formation in these galaxies should be concentrated near the high-density regions of the expanding $H$ I shell at the inner edge of the bubble. This is consistent with theories of sequential star formation (e.g., Gerola \& Seiden 1978; Gerola, Seiden, \& Schulman 1980), in which the compression associated with the shell triggers the star formation process. If the supernova-driven scenario holds, then we would expect star formation episodes to be not only correlated in age, but also in spatial distribution, along the $\mathrm{H}$ I shell structures.

Limited, low-resolution optical data tend to suggest that this scenario is possibly taking place: $\mathrm{H} \alpha$ appears concentrated on the edges of the nearly circular H I shells in Holmberg II (Puche et al. 1992; Hodge, Strobel, \& Kennicutt 1994b), Holmberg I (Westpfahl \& Puche 1994), and IC 2574 (Martimbeau, Carignan, \& Roy 1994). Another, more local, example where this also may be occurring is constellation III and the supershell LMC 4 in the LMC (Dopita, Mathewson, \& Ford 1985; Grebel \& Brandner 1998; although see Reid, Mould, \& Thompson 1987; Braun et al. 1997; Olsen et al. 1997). In addition, Puche et al. (1992) claim that broadband imaging shows the possible presence of stars or clusters at the centers of some of the largest shells in Holmberg II, which could be the populations associated with the supernovae progenitors, which remain long after the explosions.

One of the galaxies Puche \& Westpfahl (1994) sampled was Sextans A (DDO 75, UGC 205, A1008-04), a low surface brightness dwarf irregular galaxy with angular diameter of only $D_{25} \sim 4.4$ (Ables 1971; Tully 1988) and with an oddly square, or, more appropriately, diamond shape. A large number of resolved stars can be seen on a number of published ground-based (and space-based) images of the galaxy. The galaxy is seen nearly face-on (inclination $i \sim 36^{\circ}$, Skillman et al. 1988; to $45^{\circ}$, Graham \& Westpfahl 1998). A recent estimate of the distance to Sextans A by Sakai, Madore, \& Freedman (1996, hereafter SMF), based on the tip of the red giant branch (TRGB) brightness, is $1.42 \mathrm{Mpc}(m-M=25.74)$, placing it near the edge of the Local Group. At this distance, the angular diameter corresponds to a linear diameter of only $\sim 1.8 \mathrm{kpc}$ for the galaxy. The galaxy has $B_{T}=11.93$ and $(B-V)_{T}=0.30$ (somewhat bluer than the mean for Magellanic irregular galaxies; Ables 1971); at the TRGB distance modulus, $M_{B_{T}}=-13.81$. The morphology of Sextans A in $H_{I}$ had been initially studied by Skillman et al. (1988). Skillman et al. estimate a gas mass of $M \sim 5.8 \times 10^{7} M_{\odot}$ and a total mass of $M \gtrsim 1.6 \times 10^{8} M_{\odot}$.

From higher resolution and greater sensitivity maps, Puche \& Westpfahl (1994) found that Sextans A looks similar in H I morphology to other small dwarf irregular galaxies, such as Holmberg I and M81dwA (Westpfahl \& Puche 1994), in that one $\mathrm{H}$ I hole appears to dominate the overall structure, although Sextans A is a slightly faster rotator than these other small dwarfs $\left(v_{\text {rotmax }} \simeq 30 \mathrm{~km} \mathrm{~s}^{-1}\right.$ for Sextans A, compared with $\sim 15 \mathrm{~km} \mathrm{~s}^{-1}$ for Ho I and $\sim 5$ $\mathrm{km} \mathrm{s}^{-1}$ for M81dwA; Puche \& Westpfahl 1994), consistent with the central large cavity appearing to have undergone some shearing and twisting.

This galaxy has also been the object of previous intense optical study, both from the ground and, recently, from space. Its resolved stellar populations have been observed in a number of different bands. Sandage \& Carlson (1982, hereafter SC82; 1985, hereafter SC85; SC85 revises the photometry presented in SC82) published an early photometric study consisting of photographic $B V$; Hoessel, Schommer, \& Danielson (1983, hereafter HSD) obtained CCD GunnThuan gri photometry; Walker (1987, hereafter, W87) published CCD $B V$ photometry of bright stars; Aparicio et al. (1987, hereafter A87) presented deep CCD UBV photometry; and SMF recently analyzed deep CCD BVI photometry. Based on their deep $U B V$ imaging data, A87 presented a history of recent star formation in Sextans A, but they considered the star formation over only quite broad regions of the galaxy. SMF, although their $B V I$ imaging was quite deep, did not address in depth the star formation in Sextans A, dealing primarily with their distance estimate.

Recently, Dohm-Palmer et al. (1997a, 1997b) presented reports on photometric results based on deep Hubble Space Telescope (HST) imaging. Dohm-Palmer et al. (1997b) provide a detailed description of the star formation history in Sextans A over the last $1 \mathrm{Gyr}$ and illustrated the correlation of the history both in time and in space. Their results are unhampered by the problems of crowding and blending that beset ground-based studies, and the number of stars that they can statistically analyze is truly formidable.

Based on their results, Dohm-Palmer et al. (1997b) conclude that from 100-600 Myr ago, the star formation rate ( $2000 M_{\odot} \mathrm{Myr}^{-1} \mathrm{kpc}^{-2}$ ) was about 6 times larger than the rate $\left(\sim 310 \mathrm{M}_{\odot} \mathrm{Myr}^{-1} \mathrm{kpc}^{-2}\right)$ averaged over the lifetime of the galaxy and that since about 80-100 Myr ago, the rate has become remarkably higher (by a factor $\sim 20$, at $\sim 5000 M_{\odot} \mathrm{Myr}^{-1} \mathrm{kpc}^{-2}$ ) and more densely concentrated than in the past; this suggests that some unusual event has occurred in the past $100 \mathrm{Myr}$ to trigger the recent star formation. (Their movie of star formation in Dohm-Palmer et al. 1998 seems to indicate that the trigger might have begun as early as $\sim 350 \mathrm{Myr}$ ago.)

Dohm-Palmer et al. speculate on possible scenarios for this mysterious event, including a tidal interaction with neighboring Sextans B; however, they point out that these two galaxies may be separated by nearly $300 \mathrm{kpc}$, a distance over which the dynamical effects would be minimal. The trigger could also be the recent onset of a bar potential, which Skillman et al. (1988) suggest possibly exists in Sextans A, based on their data; although Puche disagrees, D. J. Westpfahl (1996, private communication) states that the gas kinematics based on their recent $\mathrm{H}$ i data could also indicate the presence of a bar. Graham \& Westpfahl (1998) suggest a possible merger or accretion event. Finally, the supernova-induced star formation mechanism could be the trigger for recent star formation. Combinations of one or more of these scenarios is, of course, also possible.

In this paper, we present deep $U B V(R I)_{\mathrm{C}}$ imaging of Sextans A, in which a large number of stars are resolved. Through our ground-based five-band imaging we have sampled nearly the entire main body of the optical galaxy, 
and therefore we can more completely describe the temporal and spatial history of star formation over a large area, particularly for the last $100 \mathrm{Myr}$, and (with far less certainty) back to about 1 Gyr. Furthermore, we examine the correlation of the recent star formation history with both the neutral $\mathrm{H} \mathrm{I}$ and the ionized gas components.

\section{DATA}

\subsection{Observations and Reductions}

The observations were conducted at the Kitt Peak National Observatory (KPNO) $2.1 \mathrm{~m}$ telescope in $U B V(R I)_{\mathrm{C}}$ on $1994 \mathrm{Feb} 11 \mathrm{UT}$, as part of a program to image several dwarf irregular galaxies showing significant structure in $\mathrm{H}$ I. The KPNO "T1KA" $1024^{2} \mathrm{CCD}$ was used in direct imaging mode at $f / 7.5$; the pixel scale was 0.31 , resulting in a field size of 5'.2. An area of Sextans A with diameter $\sim 5.3$ could therefore be imaged on the chip. All images exhibited large numbers of resolved stars in the galaxy. Total exposures in each band were divided into three separate exposures, dithering the telescope slightly between exposures to reduce pixel-to-pixel and positiondependent effects on stellar profiles and magnitudes. Total exposure times were $1800 \mathrm{~s}$ in each of the five filters. Unfortunately, although the seeing was good $\left(\sim 1^{\prime \prime} 1\right)$, the night was not photometric (nor was the entire run).

Observations were subsequently made on 1995 April 24 UT using the Lick Observatory Nickel $1.0 \mathrm{~m}$ Telescope in $B V(R I)_{\mathrm{C}}$ under photometric conditions for the purpose of calibration. The Nickel Telescope imaging system, consisting of an Orbit $2048^{2}$ CCD (binned $2 \times 2$ ), with pixel scale 0 .'37 and field size 6.3, however, has essentially no response in $U$. Fortunately, a service observing campaign (1996 April 1-5 UT) was undertaken during bright time at the KPNO $0.9 \mathrm{~m}$ telescope by R. Young Shuping to provide calibration observations for previous KPNO imaging programs conducted under nonphotometric conditions. Shuping obtained $U B V(R I)_{\mathrm{C}}$ images for us. Shorter $(600-900 \mathrm{~s})$ exposures of Sextans A were made during both sets of calibration observations.

In addition, we obtained $\mathrm{H} \alpha+[\mathrm{N} \mathrm{II}]$ and $R$-band images of Sextans A at the old KPNO "No. 1 " $0.9 \mathrm{~m}$ telescope in 1989 May 31 UT. These observations employed the old "RCA3" $508 \times 312$ chip, with pixel scale 0 ". 86 and field size 7.3 $\times$ 4.5. Three individual images through a narrowband filter with central wavelength $6563 \AA$ (bandpass $75 \AA$ ) were made. The $R$-band image taken during that observing run had an exposure time of $300 \mathrm{~s}$.

The usual bias and flat-field images were obtained during all observing runs. All image processing was performed using IRAF. ${ }^{2}$ As mentioned above, the separate $2.1 \mathrm{~m}$ exposures of Sextans A were combined into a single total image using cosmic-ray rejection for each filter. In addition, the three $\mathrm{H} \alpha+[\mathrm{N} \mathrm{II}]$ images were combined into a single 1500 s image.

A continuum-subtracted $\mathrm{H} \alpha+[\mathrm{N} \mathrm{II}]$ image was produced using the combined narrowband "on-line" observation and the broadband $R$ observation, in which $R$ was employed as the "off-line" filter, assuming that $\mathrm{H} \alpha+[\mathrm{N}$ II $]$

\footnotetext{
${ }^{2}$ IRAF is distributed by the National Optical Astronomy Observatories, which are operated by the Association of Universities for Research in Astronomy, Inc., under cooperative agreement with the National Science Foundation.
}

are the only emission lines dominating the $R$ bandpass (see Waller 1990). The scale factor for the subtraction was derived from the instrumental photometry of the field stars in common between the two sets of images. The subtracted image was not flux calibrated.

\subsection{Photometry Methods}

The DAOPHOT and ALLSTAR (Stetson 1987; Stetson, Davis, \& Crabtree 1990) routines in IRAF were used to derive the magnitudes of the resolved stars on all the $U B V(R I)_{\mathrm{C}}$ images made of Sextans A (including those made with the KPNO $2.1 \mathrm{~m}$, the Lick $1 \mathrm{~m}$, and the KPNO $0.9 \mathrm{~m}$ telescopes). These routines perform multiple fittings of a semiempirical point-spread function (PSF) to the individual stellar profiles. The PSF was established by assuming a Moffat analytical function, plus a table of residuals, using a number $(\sim 30)$ of well shaped stars on each image. Figure 1 shows the residuals of the fitting, $\sigma$, given by ALLSTAR for all stars in each band on the images made at the KPNO 2.1 $\mathrm{m}$ telescope (after elimination of probable foreground stars, obviously blended stars, and erroneous detections; see $\S 3$ below). These $\sigma$ values are representative of the internal errors in the photometry presented in this paper.

Our Lick $1 \mathrm{~m}$ telescope observations and Shuping's KPNO $0.9 \mathrm{~m}$ telescope observations not only consisted of images of Sextans A, but also images of a number of standard-star fields from Landolt (1992) spanning a wide range of colors. The standard-star instrumental magnitudes were determined through large (radius $\sim 7^{\prime \prime}$ ) synthetic apertures, which significantly included the wings of the stellar profiles, and sky annuli with radii larger than the apertures. The photometric transformations to the absolute system were computed using these instrumental magnitudes.

The ALLSTAR PSF-fitting magnitudes for stars in Sextans A imaged during the two sets of calibration observations were corrected to synthetic aperture magnitudes, using the aperture growth-curve method (Stetson 1990). However, this method was only reliable for the $\sim 15$ brightest stars (or fewer) on these shorter exposure images of the galaxy in each band. The ALLSTAR magnitudes from the KPNO $2.1 \mathrm{~m}$ images were, of course, not aperturecorrected, since these observations were not photometric, but had to be "bootstrapped" to the absolute system using the observations of Sextans A during the calibration runs.

\subsection{Transformation to the Standard Johnson-Cousins System}

For the Lick calibration run, an adequate number (30) of standard stars were observed over a large range in air mass. The aperture magnitudes of the standard stars were then used to determine the atmospheric extinction in each band and the transformation to Johnson-Cousins magnitudes. The transformation equations, derived using the PHOTCAL tasks in IRAF for the Lick Nickel $1 \mathrm{~m}$ run are

$$
\begin{aligned}
b=B & +(5.234 \pm 0.051)+(0.315 \pm 0.032) X \\
& -(0.057 \pm 0.070)(B-V)-(0.008 \pm 0.040)(B-V) X,
\end{aligned}
$$

$$
\begin{aligned}
v=V & +(4.926 \pm 0.019)+(0.196 \pm 0.012) X \\
& +(0.044 \pm 0.025)(B-V)-(0.005 \pm 0.016)(B-V) X,
\end{aligned}
$$




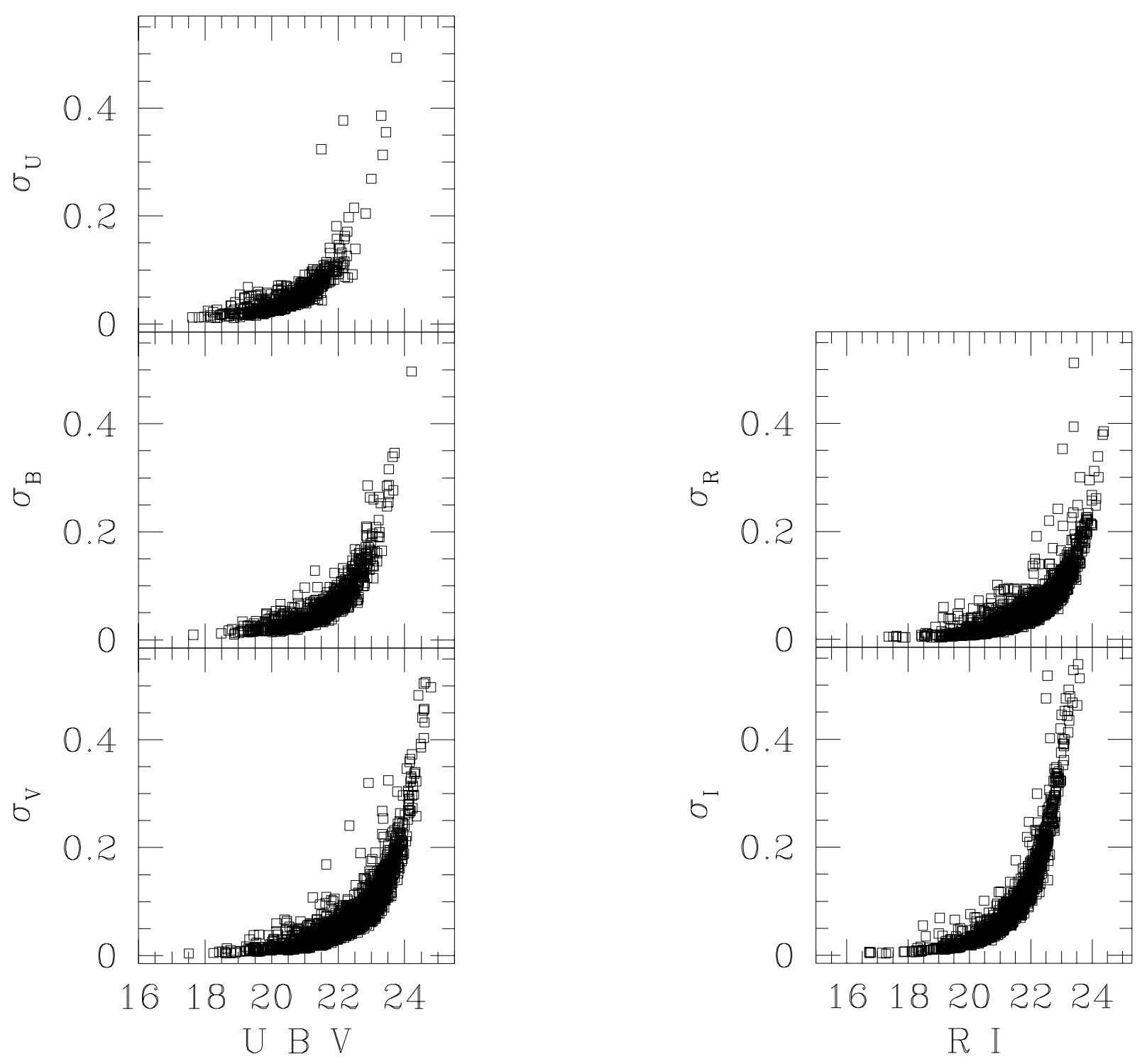

FIG. 1.-Distribution as a function of magnitude of the standard errors derived by ALLSTAR in DAOPHOT for the $U B V(R I)_{\mathrm{C}}$ bands

$$
\begin{aligned}
r= & R_{\mathrm{C}}+(4.858 \pm 0.029)+(0.134 \pm 0.019) X \\
& +(0.035 \pm 0.070)\left(V-R_{\mathrm{C}}\right)+(0.001 \pm 0.042)\left(V-R_{\mathrm{C}}\right) X,
\end{aligned}
$$

$$
\begin{aligned}
i=I_{\mathrm{C}} & +(4.908 \pm 0.053)+(0.112 \pm 0.029) X \\
& +(0.069 \pm 0.127)(R-I)_{\mathrm{C}}-(0.049 \pm 0.070)(R-I)_{\mathrm{C}} X
\end{aligned}
$$

where the lowercase letters represent the aperture (instrumental) magnitudes, the uppercase letters represent the true Johnson-Cousins magnitudes, and $X$ is the air mass of the observation. The formal errors in the coefficients are also given.

Although standard stars over a large range of color were observed during Shuping's photometric night on the KPNO 0.9 m, a smaller number (17) of stars were observed without adequately sampling over air mass. As a result, we had difficulty achieving a reasonable solution for the photometric coefficients, while allowing them all to be free vari- ables. We therefore chose to fix the air mass corrections to the nominal values appropriate for KPNO, as given in the Direct Imaging Manual for Kitt Peak (Massey et al. 1997). The transformation equations are then

$$
\begin{aligned}
u= & U+(6.972 \pm 0.030)+0.500 X-(0.076 \pm 0.248) \\
& \times(U-B)-(0.055 \pm 0.167)(U-B) X \\
b= & B+(4.902 \pm 0.007)+0.250 X-(0.019 \pm 0.039) \\
& \times(B-V)-(0.064 \pm 0.028)(B-V) X \\
v= & V+(4.665 \pm 0.004)+0.150 X+(0.134 \pm 0.020) \\
& \times(B-V)-(0.095 \pm 0.013)(B-V) X \\
r= & R_{\mathrm{C}}+(4.579 \pm 0.007)+0.100 X+(0.115 \pm 0.064) \\
& \times\left(V-R_{\mathrm{C}}\right)-(0.124 \pm 0.047)\left(V-R_{\mathrm{C}}\right) X \\
i= & I_{\mathrm{C}}+(5.353 \pm 0.007)+0.070 X+(0.005 \pm 0.058) \\
& \times(R-I)_{\mathrm{C}}-(0.082 \pm 0.044)(R-I)_{\mathrm{C}} X,
\end{aligned}
$$


TABLE 1

Comparison With Photometry from Previous StUdies

\begin{tabular}{|c|c|c|}
\hline Authors & Source & Difference \\
\hline \multicolumn{3}{|l|}{$V:$} \\
\hline HSD ...... & CCD gri & $0.350 \pm 1.024$ \\
\hline SC $85 \ldots \ldots$ & Photographic $B V$ & $0.533 \pm 0.711$ \\
\hline A87 & CCD $U B V$ & $-0.025 \pm 0.494$ \\
\hline W87 & CCD $B V$ & $0.013 \pm 0.098$ \\
\hline SMF ....... & CCD $B V I$ & $0.035 \pm 0.031$ \\
\hline \multicolumn{3}{|l|}{$B-V:$} \\
\hline HSD. & CCD gri & $-0.140 \pm 0.516$ \\
\hline SC85 ...... & Photographic $B V$ & $0.185 \pm 0.251$ \\
\hline A87 ........ & CCD $U B V$ & $-0.125 \pm 0.443$ \\
\hline W87 ...... & $\mathrm{CCD} B V$ & $0.042 \pm 0.082$ \\
\hline \multicolumn{3}{|l|}{$U-B$} \\
\hline A87. & CCD $U B V$ & $0.076 \pm 0.438$ \\
\hline \multicolumn{3}{|l|}{$V-I:$} \\
\hline SMF ...... & CCD $B V I$ & $0.046 \pm 0.054$ \\
\hline \multicolumn{3}{|l|}{$R-I:$} \\
\hline HSD .... & CCD gri & $0.093 \pm 0.198$ \\
\hline
\end{tabular}
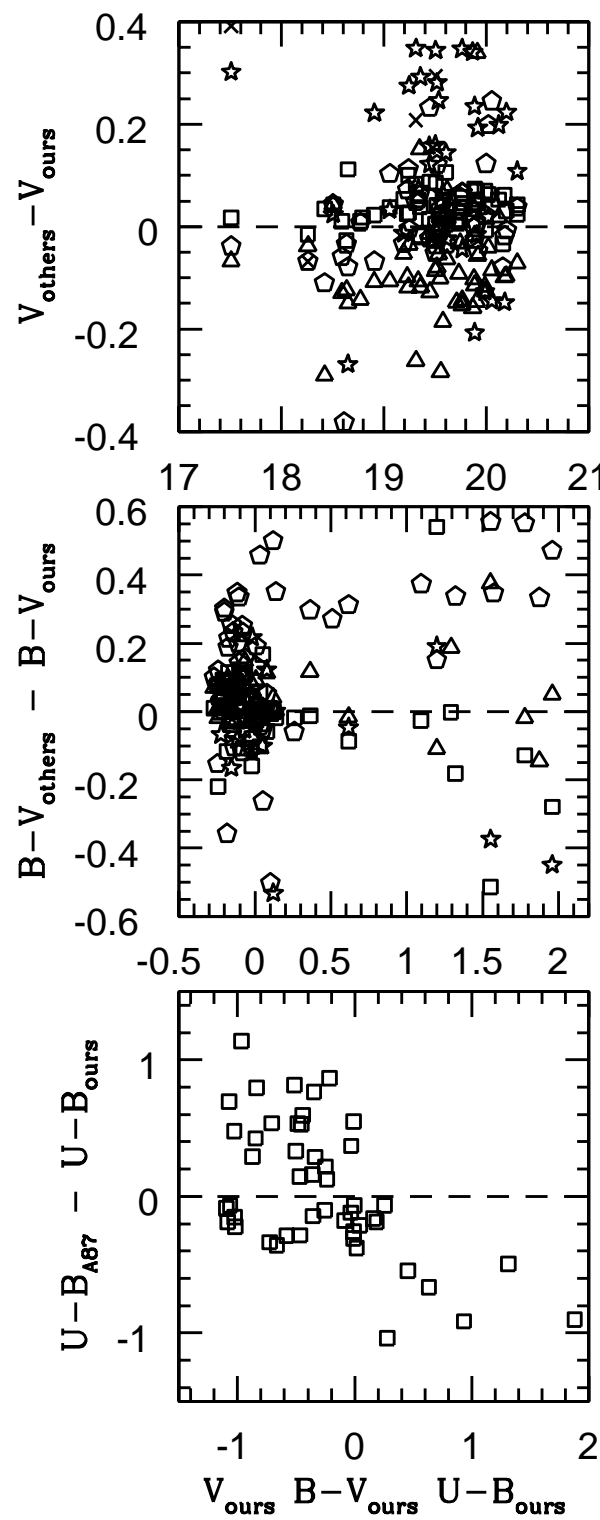

where the symbols are as above. The formal are also given for those coefficients treated as free parameters.

The aperture-corrected ALLSTAR magnitudes for the stars resolved on the images of Sextans A made during the Lick $1 \mathrm{~m}$ and KPNO $0.9 \mathrm{~m}$ runs were then transformed to the Johnson-Cousins system using the PHOTCAL routines within IRAF. We compared the final photometry results from the Lick $1 \mathrm{~m}$ and KPNO $0.9 \mathrm{~m}$ observations for stars in Sextans A in common between the two sets (we were limited to a comparison for only the brightest stars, i.e., those with $V \lesssim 17)$ and found that the $B V R I$ magnitudes and colors agreed between the two sets to the following degree: $\quad-0.003 \pm 0.024 \quad(V), \quad 0.013 \pm 0.033 \quad(B-V)$, $-0.019 \pm 0.014(\bar{V}-R)$, and $0.000 \pm 0.025(R-I)$ (the sense of the sign is "KPNO $0.9 \mathrm{~m}-$ Lick $1 \mathrm{~m}$ "). One can see that the agreement is quite good. We therefore used the photometry from the Lick run to transform the ALLSTAR magnitudes obtained from the deep KPNO $2.1 \mathrm{~m}$ imaging in $B V(R I)_{\mathrm{C}}$.
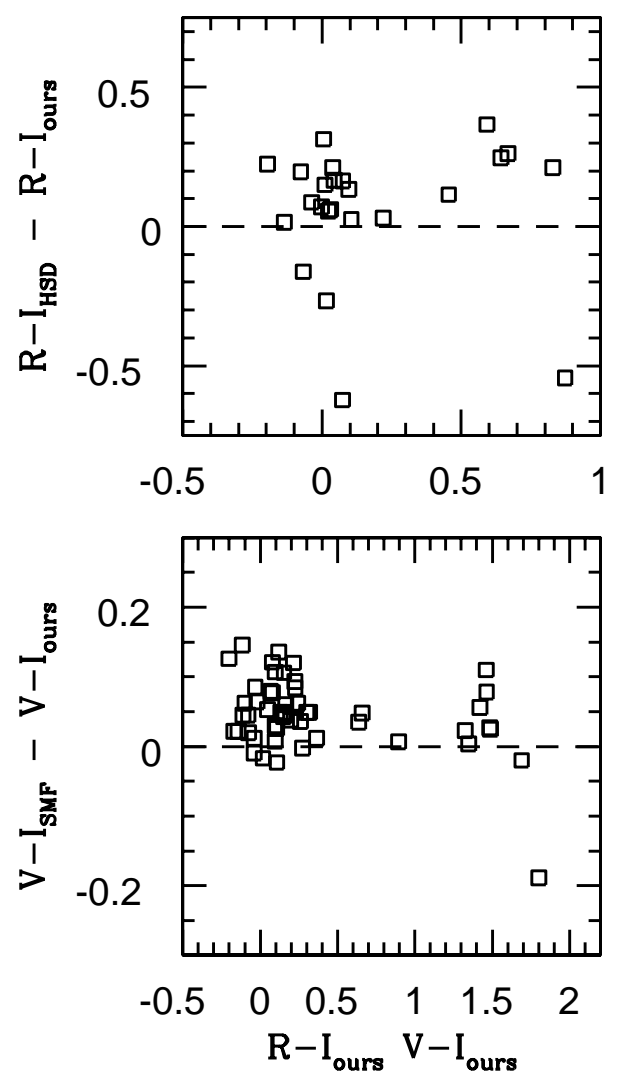

Fig. 2.-Comparison of our photometry with the photometry from other investigators in the various bands. See text for author abbreviations. $V$ is represented by squares (SMF), triangles (A87), pentagons (W87), stars (SC85), and crosses (HSD). $B-V$ is represented by squares (A87), triangles (W87), pentagons (SC85), and stars (HSD). $U-B$ is represented by squares (A87). $R-I$ is represented by squares (HSD). $V-I$ is represented by squares (SMF). 
We had no choice for $U$; the agreement between the $B V(R I)_{\mathrm{C}}$ calibration obtained from the Lick $1 \mathrm{~m}$ and KPNO $0.9 \mathrm{~m}$ imaging provided us with confidence that the $U$ calibration done on the KPNO $0.9 \mathrm{~m}$ was reasonable. We therefore transformed the $U$ photometry from the KPNO $2.1 \mathrm{~m}$ images using the photometry from the KPNO $0.9 \mathrm{~m}$ image.

\subsection{Comparison with Previous Photometry}

In any study of this kind, it is always appropriate to compare the new photometry with older published photometry when available. Fortunately, as mentioned above, Sextans A has been the subject of a good number of recent optical studies. Here, we can compare subsets of our photometry with the results from SC82, SC85, HSD (their gri magnitudes were transformed into Johnson $B V R I$ magnitudes via the transformations in Hoessel \& Melnick 1980 and Wade et al. 1979), W87, A87, and SMF. (Dohm-Palmer et al. 1997a and 1997b present BVI photometry using HST, but they do not provide their data in tabular form, so we do not make a comparison with their photometry.)

The stars that have been most used as the basis of comparison in all these previous studies are the bright stars listed in SC82. In Table 1, we list the mean differences of our measurements for the various colors for the SC82 stars with the measurements from these other studies. In Figure 2, we show a comparison of our magnitudes and colors for the SC82 stars with those obtained by the other studies.

One can see that our $V$ magnitudes are in very good agreement with those of SMF, A87, and W87 but differ substantially from SC85 and HSD, the former likely because of the zero-point problems associated with the photographic photometry, and the latter likely because of the uncertainty in the transformation from the Gunn-Thuan system to the Johnson system, as noted by SMF. Our $B-V$ colors agree very well with those in W87, but less so with those from the other studies. Our $U-B$ colors are in reasonably good agreement with those from A87, but in the case for both $U-B$ and $B-V$, we point out the large amount of scatter for even the brightest stars studied in A87.

One can see very good agreement of our $V-I$ colors with those from SMF, with an offset of $\sim 0.05$ between our colors and theirs (although the rms in the comparison is roughly equivalent to this possible offset; in addition, we notice a similar offset between the $I$ magnitudes from SMF and those from Dohm-Palmer et al. 1997a in the latter's Fig. 7 for $I \lesssim 20.3$ ). Finally, we compare our $R-I$ (Cousins system) colors with the $R-I$ (Johnson system) colors from HSD. The offset of $\sim 0.1$ agrees with the zero point of 0.12 in the transformation from the Johnson to the KronCousins system derived using asymptotic giant branch stars by Costa \& Frogel (1996).

In all, we believe the agreement of our photometry with previous photometry to be quite satisfactory.

\subsection{Transformation to Absolute Coordinates}

Absolute star positions were derived by converting the pixel centroids of the stars on our KPNO $2.1 \mathrm{~m}$ images from ALLSTAR into absolute coordinates using the Guide Star Astrometric Program (GASP) software in STSDAS/
TABLE 2

Comparison of Actual and Predicted Foreground Star Counts

\begin{tabular}{cccc}
\hline \hline$V$ & $B-V<0.8$ & $0.8<B-V<1.3$ & $1.3<B-V$ \\
\hline Actual counts: & & & \\
$18 \ldots \ldots$ & 8 & 1 & 4 \\
$20 \ldots \ldots$ & 196 & 20 & 40 \\
$22 \ldots \ldots$ & 725 & 209 & 83 \\
$24 \ldots \ldots$ & 55 & & 54 \\
Predicted counts: & & 1.4 & \\
$18 \ldots \ldots$ & 1.1 & 1.1 & 1.9 \\
$20 \ldots \ldots$ & 2.4 & 2.4 & 5.5 \\
$22 \ldots \ldots$. & 2.4 & 2.8 & 10.8 \\
$24 \ldots \ldots$ & 0.9 & & 17.7 \\
\hline
\end{tabular}

${ }^{\text {a }}$ Based on interpolating the model counts from Ratnatunga \& Bahcall (1985) for the field appropriate for Sextans A.

IRAF and the Digitized Sky Survey ${ }^{3}$ image of the Sextans A field obtained from the Canadian Astronomy Data Center (CADC), from which absolute coordinates for stars on the Palomar Observatory Sky Survey can be measured. The accuracy obtained in these absolute positions by this technique $\left(\sim 1^{\prime \prime}-2^{\prime \prime}\right)$ is adequate for our comparison with the $\mathrm{H} \mathrm{I}$ radio map, given the relatively lower resolution of the radio data (see $\S 4)$.

\section{ANALYSIS OF THE PHOTOMETRY}

We present here a brief description of our photometric results. We have removed obvious foreground stars from our star list, e.g., the notoriously bright red foreground star in the northeast quadrant of Sextans A, and those stars that A87 and SC82 previously identified as foreground stars (e.g., those that SC82 measured as proper-motion stars). These are essentially all stars with $V \lesssim 17.5$.

We can estimate how many foreground stars fainter than this $V$ should be in our field, from the Ratnatunga \& Bahcall (1985) model for the galactic coordinates of Sextans A (we interpolate the model appropriate for Sextans A from the nearby fields including the galaxies Pal 3 and Leo I). In Table 2 we list, for our $27 \mathrm{arcmin}^{2}$ field, the predicted number of stars in three color bins and four magnitude bins, along with the estimated number of actual stars found within these bins. Of course, without identification spectra for individual stars, we have no means of eliminating possible foreground interlopers from our star list, but the comparison in Table 2 should be kept in mind throughout the paper.

Finally, we have also used our color-color diagrams (§ 3.2) to isolate and remove stars from our list that clearly have grossly discordant colors, indicating possible blends, unresolved nebulosity, or other photometric errors. We have eliminated erroneous detections along the two bad rows on the combined images and within the saturated

\footnotetext{
${ }^{3}$ The Digitized Sky Surveys were produced at the Space Telescope Science Institute (STScI) under US government grant NAGW-2166. The images of these surveys are based on photographic data obtained using the Oschin Schmidt Telescope on Palomar Mountain and the UK Schmidt Telescope. The places were processed into the present compressed digital form with the permission of these institutions. The National Geographic Society-Palomar Observatory Sky Atlas (POSS-I) was made by the California Institute of Technology with grants from the National Geographic Society.
} 
image of the aforementioned bright red foreground star; we have also removed stars that are too close to the edge of the CCD in any band to provide reliable photometry.

We arrive at 2525 stars detected in $V$ and at least one other band. Because of space limitations, we do not list the magnitudes and colors for all the stars here. An electronic tabulation of magnitudes, colors, and absolute positions of the stars can be obtained courtesy of the CDS.

\subsection{Luminosity Functions}

We can derive a luminosity function (LF) for the stars in the galaxy and compare it with those for other dwarf irregular galaxies. In Figure 3, we show the global differential LF for all detected stars in each of the bands. Crowding and blending of stars in our images limit the depth and accuracy of the photometry we have obtained. This can be represented by the completeness of our photometry. One can see that incompleteness significantly affects the photometry in each of the bands at a given magnitude. Our photometry is most complete in the $V$ band, then most complete in $R$, then $I, B$, and finally, $U$. The photometry is seriously affected by incompleteness at $V \sim 23, R \sim 22.5, I \sim 22, B \sim 21.7$, and $U \sim 20.7$.

We also derive the LF for only the main-sequence stars. Following the recommendation by Freedman (1985), we separate these stars from the evolved "blue-loop" supergiants (which are indistinguishable from the main-sequence stars in the blue plume on the $(V, B-V)$ CMD in Fig. 5 below) by selecting those stars with $U-V \lesssim-1.05$ (we use a similar criterion in $U-B$ in $\S 3.3$ to distinguish the hot main-sequence stars). In Figure 3, we show the differential LF for only those 241 stars ( filled circles). A least-squares fit to the data for the main-sequence stars brighter than $V=22$ (fainter than which incompleteness clearly affects our statistics) results in a slope, $d \log N / d V=0.48$.

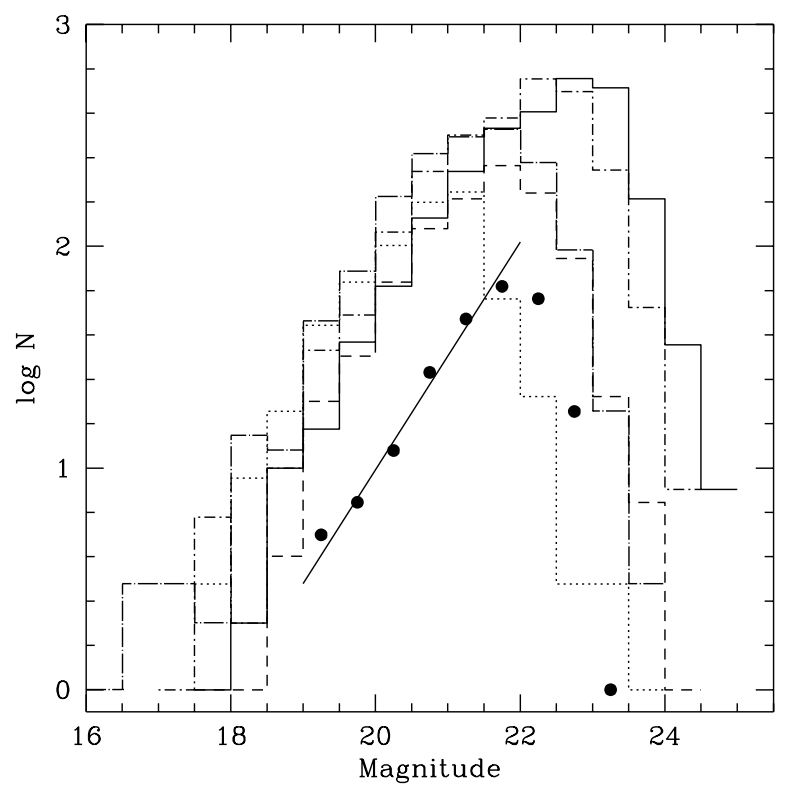

FIG. 3.-Differential LFs for Sextans A in $U$ (dotted line), $B$ (dashed line), $V$ (solid line), $R$ (short dashed-dotted line) and I (long dashed-dotted line) for all detected stars. Also shown is the function for only mainsequence stars ( filled circles), i.e., those with $U-V \lesssim-1.05$. The line represents a least-squares fit to the main-sequence points for stars with $V \lesssim$ 22 , resulting in a slope $d \log N / d V=0.48$, which is consistent with that found for other dwarf irregular galaxies and also with the functions for all stars in all the bands.
This is consistent with the slope for other dwarf irregular galaxies, such as WLM, Sextans B, and NGC 6822 (see, e.g., Tosi et al. 1991; Greggio et al. 1993; Marconi et al. 1995) and implies that Sextans A has a star formation rate and initial mass function that are not unlike other dwarf irregular galaxies studied so far. As pointed out by Hunter \& Plummer (1996), Sextans A currently has a star formation rate $\left(\sim 0.006 M_{\odot} \mathrm{yr}^{-1}\right)$, when normalized to its linear scale, which is typical of other dwarf irregular galaxies (see also Hunter \& Gallagher 1986), being neither very low nor excessively high.

Interestingly enough, similar slopes are found for the LFs for all stars in each of the bands for which the photometry is complete $(0.44$ for all stars in $V ; 0.44$ in $B ; 0.53$ in $U ; 0.48$ in $R$, and 0.53 in $I$ ). This implies that the mass function averaged over more than $1 \mathrm{Gyr}$ has been relatively constant in Sextans A. We have also isolated just those stars along the putative bar in the galaxy; we find that the slope of the LF for these stars in $V$ is 0.51 , similar to the slopes in $V$ and other bands for all stars in Sextans A.

\subsection{Color-Color Diagrams}

In Figure 4, we present the color-color diagrams for Sextans A. The solid line in each diagram shows the location of the (unreddened) main-sequence, giant, and supergiant branches, based on theoretical models (see $\S 3.4$ ). We have indicated in these color-color diagrams the direction of the reddening vector. We also show representative uncertainties in the observed colors. On the $(U-B, B-V)$ diagram we can see two clumps of stars, the bluer mainsequence stars and the somewhat redder blue supergiants. On the $(B-V, V-I)$ diagram, we again see a blue clump of stars, which is a mixture of main-sequence stars and supergiants and a smaller clumping of red supergiant stars. The general agreement between the model tracks and the observed color distributions indicates that the amount of reddening to and within the galaxy must be in general small (see § 3.3). But, some reddening is clearly necessary, based on these diagrams, on the amount of Galactic foreground reddening, and on the results of other investigators from colors of individual stars (e.g., SMF). The reddening also appears somewhat variable.

\subsection{Color-Magnitude Diagrams}

The most direct information on the stellar populations and relative star formation histories in galaxies can be obtained from the analysis of color-magnitude diagrams (CMDs) based on deep photometry of the resolved populations. In this paper we have observed at five optical bands: our bluest colors can very adequately trace out the youngest, hottest stars in the galaxy, while, as Aparicio \& Gallart (1995) have demonstrated in their study of the Pegasus dwarf galaxy, photometry in red colors is also necessary to derive information about stars of all ages.

In Figure 5 we present the CMDs for Sextans A in the various colors based on our photometry. What is immediately evident from examining all of the CMDs, as has been found in previous studies of Sextans A, is the rich and various array of populations of different ages, from mainsequence stars to red supergiants to asymptotic giant branch (AGB) stars. Such populations have been identified in this and other dwarf galaxies from the ground, but never before with this color baseline. 


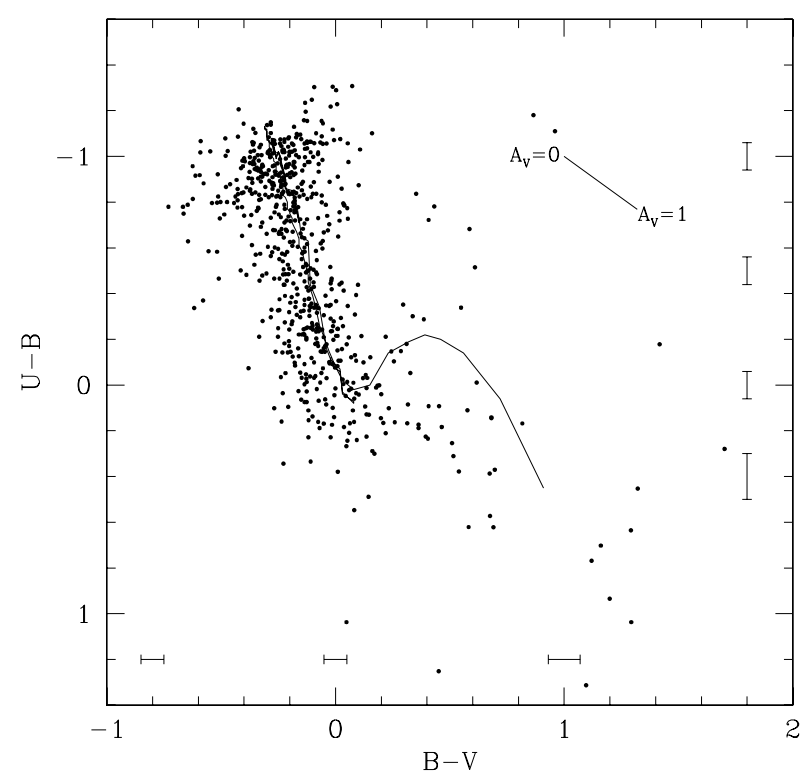

FIG. $4 a$

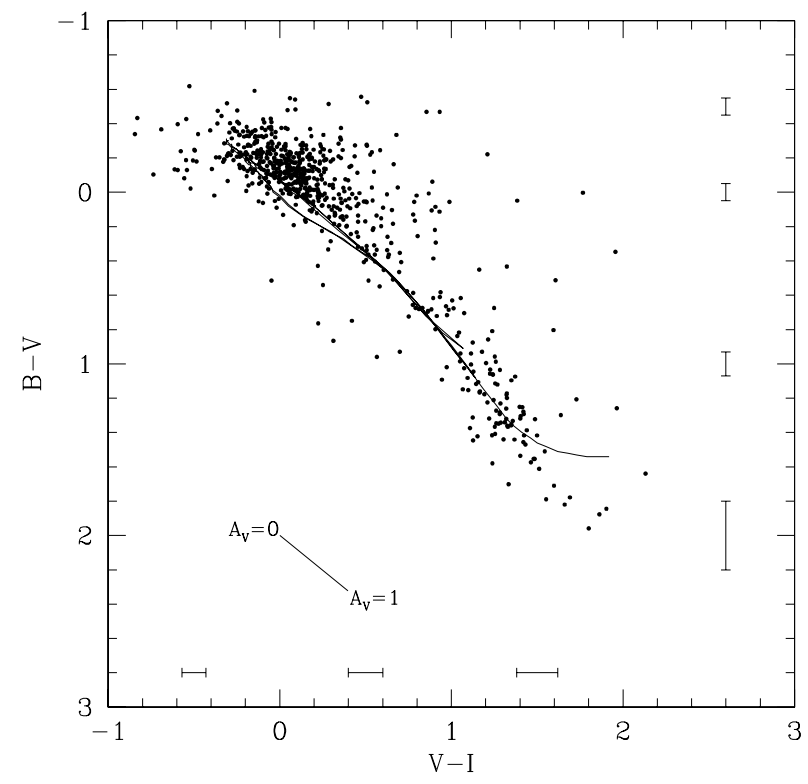

FIG. $4 b$

Fig. 4. - Color-color diagrams for Sextans A. In $(a)$ we show the $(U-B, B-V)$ diagram, and in $(b)$ we show the $(B-V, V-I)$ diagram. For each diagram we show the unreddened $4 \mathrm{Myr}$ isochrone from Bertelli et al. (1994) with metallicity $Z=0.001$; for $(b)$ we also show the 100 Myr isochrone (note the poorer agreement of the AGB stars with the model). For both figures we also show representative uncertainties in the colors and the direction of the reddening vector.

The practical detection limit for our photometry is $V \lesssim$ 23. In our deep ground-based images, a diffuse background of faint stars in the galaxy is also discernible below this limit.

On the $(V, B-V)$ and $(V, V-R)$ CMDs, one can clearly see the blue sequence, or "plume," of stars, with $B-V \lesssim$ $0.2(V-R \lesssim 0.3)$. The greatest limitation to separating out the various populations in Sextans A, based on our imaging is, of course, crowding and blending. Despite this limitation in resolution, however, our $(V, U-B)$ CMD clearly shows that this blue plume separates into the main sequence (with $U-B \lesssim-0.7)$, down to about spectral type B1 $(V \sim 22)$, and the evolved blue-loop supergiant stars, with $-0.7 \lesssim U-B \lesssim 0.3$, which are burning helium $(\mathrm{He})$ at their cores, although some small contamination exists from main-sequence turnoff stars. (This blue loop is not as evident in our $[V, B-V]$ and $[V, V-R]$ CMDs, in part because of the above-mentioned crowding and blending of main-sequence and evolved stars on the CCD images, but also because of the fact that $U-B$ affords a much better temperature resolution for luminous blue stars and therefore assists in overcoming the degeneracy in the blue plume.)

We therefore have been able to detect the brighter $(V \lesssim$ 22) blue-loop population based on our ground-based data, even in a galaxy as distant as Sextans A, through deep $U B V$ imaging.

One notes on the blue CMDs the very bright star with $V=17.51, U-B=-0.03$, and $B-V=0.14 ; \mathrm{A} 87$ point to it as likely being a supergiant member of Sextans A primarily because of its location in the bright blue clustering of stars in the southeast. The PSF for this object does appear stellar, so it is not likely to be a blend. For this star $M_{V} \simeq$ -8.3 for a distance modulus of 25.8 (see $\S 3.4$ ). It appears therefore to be $\sim 2$ mag brighter than the blue straggler binary stars found in the young SMC cluster NGC 330 (Grebel, Roberts, \& Brandner 1996). It is more likely to be a luminous blue variable star, similar to the one found by
Drissen, Roy, \& Robert (1997) in NGC 2366. This star clearly deserves more attention.

One can also see on the $(V, B-V) \mathrm{CMD}$, and more so with the addition of the $R$ band in the $(V, V-R) \mathrm{CMD}$, that the number of blue-loop stars is relatively larger than the number of red supergiants, in a "red plume" of stars with $B-V \gtrsim 0.5(V-R \gtrsim 0.6)$ and $V \lesssim 22$. More blue supergiants than red supergiants may exist in this galaxy. This is consistent with the fact that, at low metallicity, stars spend more time as blue supergiants than red supergiants during their He core-burning phase (Bertelli et al. 1994).

On the $(I, R-I)$ and $(I, V-I)$ CMDs the conspicuous red plume of stars are again visible at $0.8 \lesssim V-I \lesssim 1.6$ $(0.3 \lesssim R-I \lesssim 0.8)$ and $I \lesssim 22.3$, with two or three conspicuous clumps of red supergiants, as noted by SMF, at $I \sim 21.2,20.6$, and 19.8. Also visible is the broad clump of red stars at $0.8 \lesssim V-I \lesssim 1.8(0.3 \lesssim R-I \lesssim 0.8), I \sim 22$, which is the top of what Aparicio \& Gallart (1995) refer to as the "red tangle" of RGB stars, old and intermediate-age AGB stars, and intermediate-age blue-loop stars. The TRGB, although likely contaminated by intermediate-age AGB stars, is at $I \sim 21.8$ and $V-I \sim 1.3$ (SMF).

The stars fainter than $I \sim 19$ with $V-I \lesssim 2(R-I \lesssim 0.9)$ are what Aparicio \& Gallart (1995) refer to as the "red tails" of intermediate-age and old AGB stars. Aparicio \& Gallart point out that the "length" of the "tail" (i.e., how red this feature extends on the CMD) is a function of metallicity: the longer the tail, the greater the metallicity. The observed tails for Sextans A do not extend as red in color as do, for example, those on the CMDs for the Pegasus dwarf galaxy (Aparicio \& Gallart 1995), consistent with the metallicity difference between Sextans A and Pegasus (Skillman, Bomans, \& Kobulnicky 1997; Aparicio, Gallart, \& Bertelli 1997).

Of particular curiosity are the very bright red stars seen on the red CMDs. Although they appear to be especially, and possibly anomalously, bright, to be members of Sextans A, we have no particular evidence to the contrary; for 

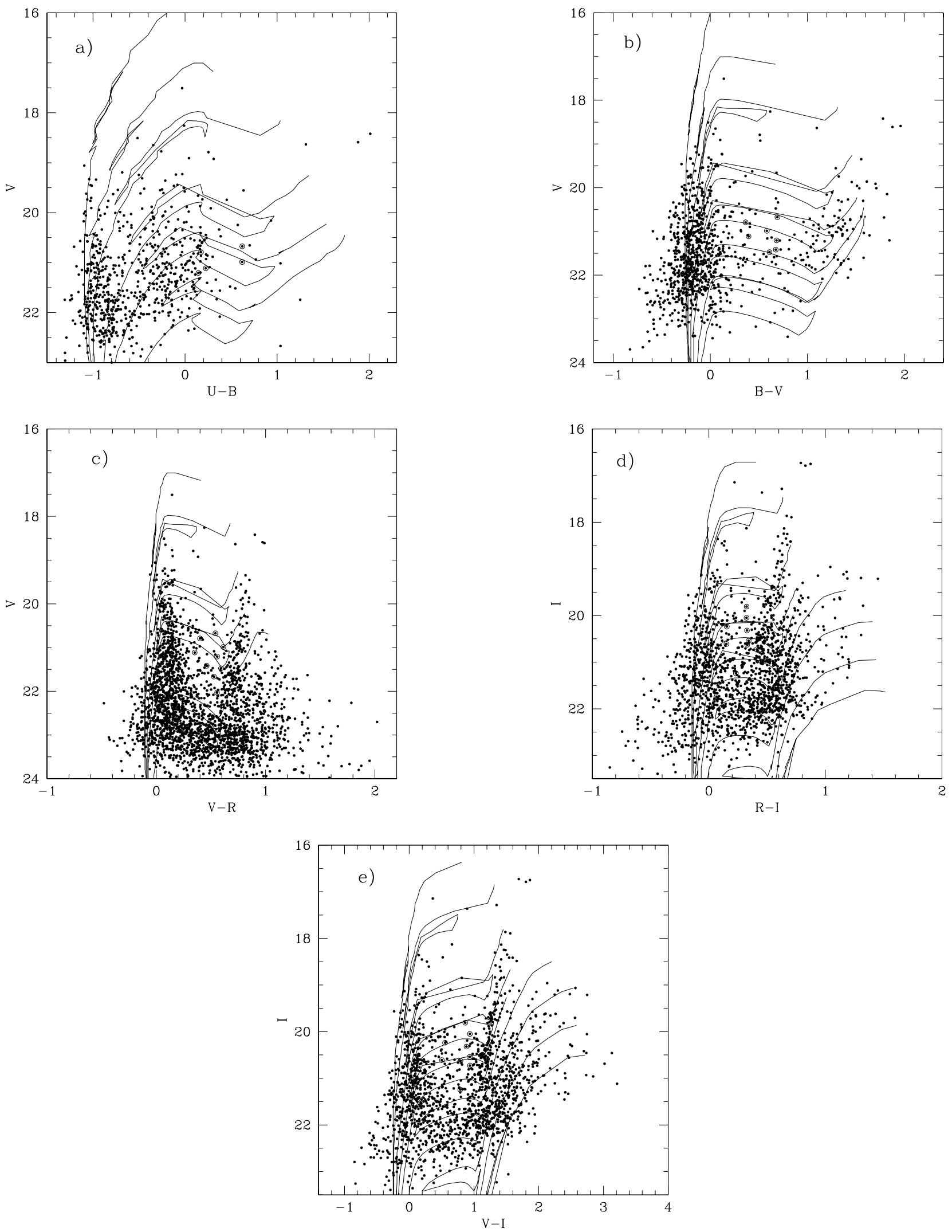

Fig. 5.-Color-magnitude diagrams for the stars in Sextans A. In (a) we have also plotted the theoretical isochrones from Bertelli et al. (1994) with metallicity $Z=0.001$ for the ages 4, 8, 12, 25, 40, and $80 \mathrm{Myr}$. In $(b)$ we also include the $125 \mathrm{Myr}$ isochrone. In $(c)$ we have plotted the isochrones for 8, 12, 25, 40 , and $125 \mathrm{Myr}$. In $(d)$ and $(e)$ we have plotted the isochrones in $(c)$, but have also included the isochrones with ages 60 , and 0.3 , 1 , and $3 \mathrm{Gyr}$. On each we show the known Cepheids (Piotto et al. 1994) with open circles surrounding the data points. A distance modulus of 25.8 mag and an average extinction of $A_{V}=0.16$ have been assumed. 
example, they are not among the proper-motion stars in $\mathrm{SC82}$. All but one of the stars is in or near a region of recent star formation. Short of having spectra for these stars, we consider them to be members of the galaxy.

We also show on all CMDs, highlighted with open circles, the magnitudes and colors of the known Cepheids from Piotto, Capaccioli, \& Pellegrini (1994).

\subsection{Stellar Population Ages}

On the CMDs in Figure 5, we have compared our photometry with a series of theoretical isochrones calculated by the Padova group (see Bertelli et al. 1994 and references therein), which we use to estimate the ages of the stellar populations in Sextans A. We have chosen those isochrones computed with a metallicity $Z=0.001$, which is consistent with that inferred from the oxygen abundance for this galaxy (Skillman, Kennicutt, \& Hodge 1989). We do not extensively sample the oldest populations, where consideration of significantly lower metallicity would be important. We have found that isochrones of higher metallicity do not match the positions of the stars in color and magnitude nearly as well as those of the chosen metallicity.

Before further comparison with the isochrones is made, it is first necessary to choose a distance to the galaxy and to consider the amount of reddening that the observed stars are experiencing in order to estimate ages for the various populations. We therefore adjust the isochrones on the CMDs accordingly.

First, we have adopted a distance modulus to Sextans A, which is a compromise between the modulus derived from the TRGB and Cepheid brightness methods given by SMF, that is, $m-M \simeq 25.8 \mathrm{mag}$ (which corresponds to a distance of $1.44 \mathrm{Mpc})$. Second, we have assumed a reddening for the galaxy of $E(B-V)=0.05$. The amount of Galactic reddening is $E(B-V)=0.02$ (Burstein \& Heiles 1984), but, as SMF point out, $E(B-V) \simeq 0.05$ is likely appropriate for the main body of the galaxy, where most of the young stars and gas are found (SMF find a higher $E[B-V]$ for the older red giant population in the galaxy). So, we have assumed this value, which, assuming a normal reddening law, i.e., $A_{V}=3.1 E(B-V)$, corresponds to $A_{V} \simeq 0.16$. Note that this is somewhat larger than the reddening assumed by Dohm-Palmer et al.(1997a, 1997b), which is essentially only due to the Galactic component. Based on our reddening assumption, we follow Cardelli, Clayton, \& Mathis (1989) in deriving the extinction for the other bands and assume that $E(U-B) \simeq 0.7 E(B-V)$.

From examining all the CMDs it is evident, regardless of their exact ages, that a number of stellar populations of various ages coexist in Sextans $\mathrm{A}$, as has been noted in previous studies (e.g., A87; Dohm-Palmer et al. 1997b). The star formation does not seem to have been continuous but instead appears to have occurred more in bursts or episodes. We do not try to execute a thorough and exact analysis of the population ages through more sophisticated means such as synthetic CMDs (e.g., Aparicio et al. 1996). Based on our more cursory analysis with the theoretical isochrones, it can be seen from the CMDs constructed from the $U, B$, and $V$ bands that the blue plume of stars consists of several young populations, from very young of roughly only a few megayears) to significantly older populations with ages $\lesssim 100 \mathrm{Myr}$. Clear gaps in stellar age within this time range exist; this is best seen in the redder populations (i.e., with $B-V \gtrsim 0.4$ ).
The redder CMDs show three noticeable clumps of red supergiants, with ages $\sim 40, \sim 60$, and $\sim 80$ Myr. The number of red supergiants both older and younger than these ages appears to be significantly smaller. These stars must have formed in three different bursts of recent star formation. The clump of RGB stars (red tangle), for which we can resolve only the top, appears to correspond to an age of $\gtrsim 100 \mathrm{Myr}$. The oldest populations we can resolve (the diffuse quantity of red stars with $V-I \gtrsim 1.5$, comprising the various red AGB tails) appear to have ages from $\sim 0.2-0.3$ Gyr to several Gyr, although we can only probe these populations with accuracy to ages 1-2 Gyr. Our photometry is not deep or complete enough to resolve stars older than this.

From our CMDs we find that the Cepheids in Sextans A have ages $\gtrsim 40 \mathrm{Myr}$ and appear to be younger than $\sim 100$ Myr.

\section{SPATIAL DISTRIBUTION OF STELLAR POPULATIONS}

First, we examine the spatial distribution of the youngest population of stars in Sextans A. We consider stars with ages $\lesssim 50$ Myr. Dohm-Palmer et al. (1997b; see their Fig. 13) have found that the star formation rate beginning $\sim 50$ Myr ago has been a factor of $\sim 20$ larger than the timeaveraged rate for the galaxy, and therefore we illustrate the extent of the most vigorous star formation over the last 1 Gyr. In Figure 6, we represent on the $V$-band image of the galaxy four young populations of stars: (1) those stars with $-1.2 \lesssim U-B \lesssim-1.0$ and $V \lesssim 22$, which are the youngest, bluest main-sequence stars (plus signs); (2) those young stars with magnitudes and colors, particularly in $U-B$, which make them likely main-sequence turnoff stars and supergiants with ages $\lesssim 12 \mathrm{Myr}$, including the very bright blue and red supergiant stars mentioned in $\S 3$ (crosses); (3) those blue He-burning stars (with $-0.7 \lesssim U-B \lesssim 0.3$ ) with $V \lesssim 20.7$, i.e., those with ages $\lesssim 50 \mathrm{Myr}$ (circles); and (4) the corresponding red He-burning stars, seen on the $(I, V-I) \mathrm{CMD}$, with $1.2 \lesssim V-I \lesssim 1.6$ and $I \lesssim 19.7$ (squares).

As Dohm-Palmer et al. (1997b) emphasize, the blue Heburning stars follow a somewhat redder, nearly vertical track parallel to the main sequence and are about 2 mag brighter than the corresponding main-sequence turnoff stars of the same age. The position in magnitude of a blue He-burning star on the CMD is determined by the star's mass and, therefore, its age. The blue He-burning stars are clearly very useful age probes for stellar populations, back to about $600 \mathrm{Myr}$ in Sextans A. This is only true at these low metallicities for blue He-burning stars with ages older than $\sim 20 \mathrm{Myr}$, or $V \gtrsim 19.3$ for Sextans A, since the Heburning phase for stars with $M>15 M_{\odot}$ is not well understood (Chiosi, Bertelli, \& Bressan 1992; Dohm-Palmer et al. 1997b).

One immediately can see that the recent star formation over the last 50 Myr has occurred in specific regions in Sextans A and is not uniformly distributed. In particular, the very young, hot, blue main-sequence stars are primarily found near surface brightness $\mu_{B} \simeq 25.9$ mag $\operatorname{arcsec}^{-2}$, or a radius $r \simeq 2.3$ (Ables 1971). As pointed out previously by HSD, A87, and others, these hot stars are primarily found in the bright clusters in the southeast and to the west. But they are not exclusively in just these two locations. Another significant clustering of these stars is to the northwest, and a small cluster is to the northeast. A smaller number of these 


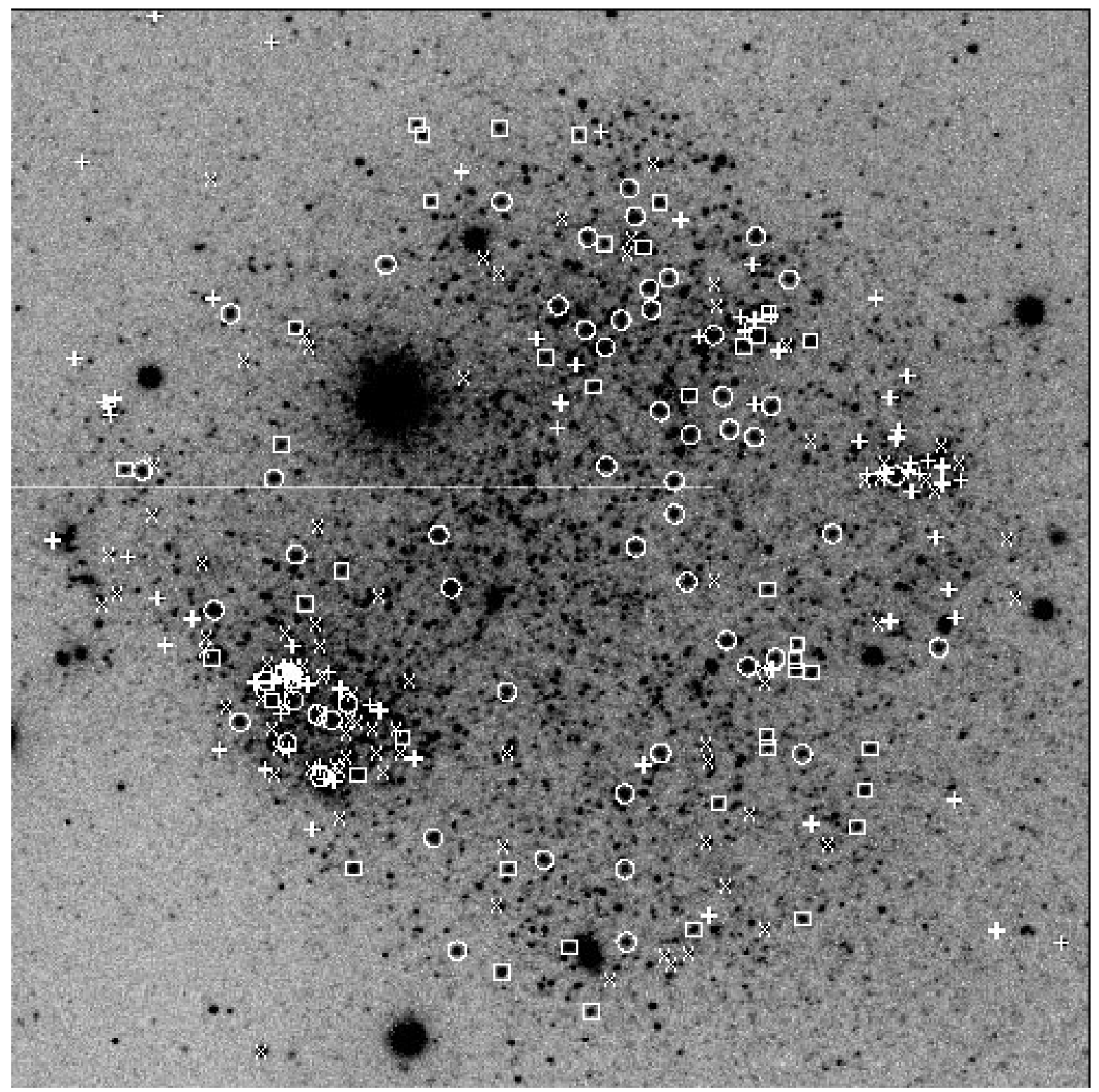

Fig. 6.-On the $V$-band image of Sextans A we show the following young populations of stars: (1) stars with $-1.2 \lesssim U-B \lesssim-1.0$ and $V \lesssim 22$, which are the youngest, bluest main-sequence stars (pluses); (2) stars with magnitudes and colors, particularly in $U-B$, which make them likely main-sequence turnoff stars and supergiants with ages $\lesssim 12$ Myr (crosses); (3) blue He-burning stars (with $-0.7 \lesssim U-B \lesssim 0.3$ ) with $V \lesssim 20.7$, i.e., with ages $\lesssim 50$ Myr (circles); and (4) the corresponding red He-burning stars, with $1.2 \lesssim V-I \lesssim 1.6$ and $I \lesssim 19.7$ (squares).

stars are found within the main optical body of the galaxy. Large areas of the galaxy clearly exist where no recent star formation has taken place.

What is most intriguing is that some of these stars are also found beyond $\mu_{B} \simeq 25.9 \mathrm{mag} \operatorname{arcsec}^{-2}\left(r \simeq 2^{\prime} .3\right)$ in the northeast and the southwest. Based on Table 2, we consider the likelihood of foreground stars in such numbers superposed on this small field to be very small. We conclude that recent star formation appears to even be occurring beyond the main optical surface brightness of Sextans A. Massive stars may form seemingly "isolated;" though this does not occur often, it is not unusual (e.g., Massey, Johnson, \& DeGioia-Eastwood 1995; Massey et al. 1995).

The bright young supergiants, not surprisingly, have a very similar spatial distribution to the very young mainsequence stars. (One supergiant star can be seen in the extreme southeast well beyond $r \simeq 2.3$; it is possible that this is a foreground star, based on Table 2, although its magnitude and color $[V=18.92, \quad B-V=0.52$, $U-B=0.31]$ are consistent with other bright supergiants seen within this radius.) But these stars, as plotted in Figure 6 , have ages up to $\sim 12 \mathrm{Myr}$, and some are therefore older than the youngest population represented (the hot main sequence). Some of these supergiants are seen in regions of the galaxy not directly associated with the youngest stars and therefore likely trace out regions of somewhat older star formation.

The evolved He-burning stars, both blue and red with ages up to $\sim 50 \mathrm{Myr}$ are also found near $r \simeq 2$ '.3, but, particularly in the west, their spatial distribution is appreciably inward, located more toward the galaxy's optical center, extending from the northwest down to the southwest. Many of these stars are also found in the bright cluster of stars in the southeast, indicating that star formation in that region has been occurring for at least $\sim 50 \mathrm{Myr}$. The brighter stars with ages $\sim 50 \mathrm{Myr}$ in this cluster appear to form a "ring," 
with radius of $\sim 300 \mathrm{pc}$. Several of these blue-loop stars are also found in the smaller cluster of stars in the northwest. But, most noticeably, only one bright blue He-burning star is found in the galaxy's second-largest star-forming region, in the west, and this star, from its position on the CMDs (with $V=19.23, B-V=0.12$, and $U-B=-0.03$ ), is possibly as young as $\sim 15 \mathrm{Myr}$. This region of star formation, which Dohm-Palmer et al. (1997a, 1997b) could not study, is clearly one of the youngest in the galaxy, along with the small clustering of hot stars in the northeast. Star formation in these regions could be as recent as $\lesssim 20 \mathrm{Myr}$.

In summary, the recent massive star formation over the last $50 \mathrm{Myr}$, which has been so pronounced in Sextans A, has been confined toward the edge of the main body of the galaxy, near $\mu_{B} \simeq 25.9$ mag $\operatorname{arcsec}^{-2}$, and may have progressed even beyond this point. In particular, in the west, it has been progressing outward from more central regions. Much of the recent star formation has been concentrated in the large clustering of stars in the southeast, and to the northwest. The youngest large concentration of star formation has been in the clustering in the west. Noticeable large holes can be seen in the distribution of young stars. No appreciable recent star formation has occurred at the optical center of the galaxy.

Next, we examine the spatial distribution of older populations in Sextans A. In Figure 7, we again show the $V$ image of the galaxy. Shown on this figure are two populations of stars: (1) those blue He-burning stars with ages 50-100 Myr, with $V \gtrsim 20.7$ to $V \sim 22$ (the faintest blue He-burning stars that we can confidently resolve; squares) and (2) the corresponding red He-burning stars with $19.7 \gtrsim$ $I \gtrsim 21.3$, with ages to $\sim 100 \mathrm{Myr}$ (circles).

The more central concentration of the distribution of the stars with ages 50-100 Myr is more noticeable than for the younger populations. Some structure and clustering also appears in the distribution of these older stars, which does not appears to be random. We have used a large age bin ( $\sim 50$ Myr wide) for these stars, so small timescale details in

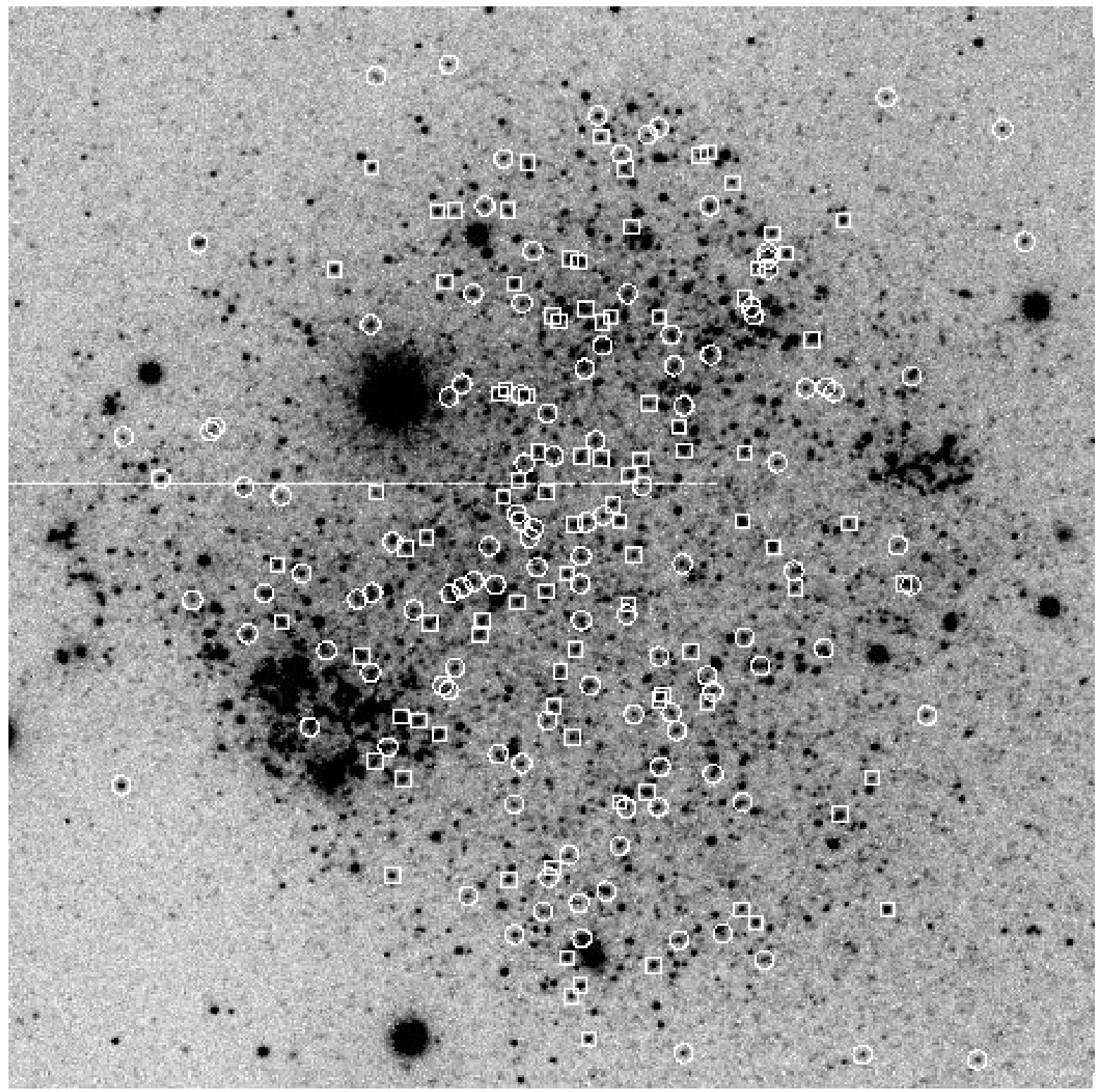

Fig. 7.-On the $V$-band image of Sextans A we show the following populations of stars: (1) those blue He-burning stars with ages $\sim 50-100$ Myr, with $V \gtrsim 20.7$ (squares); and (2) the corresponding red He-burning stars with $19.7 \gtrsim I \gtrsim 21.3$, with ages to $\sim 100 \mathrm{Myr}$ (circles). 


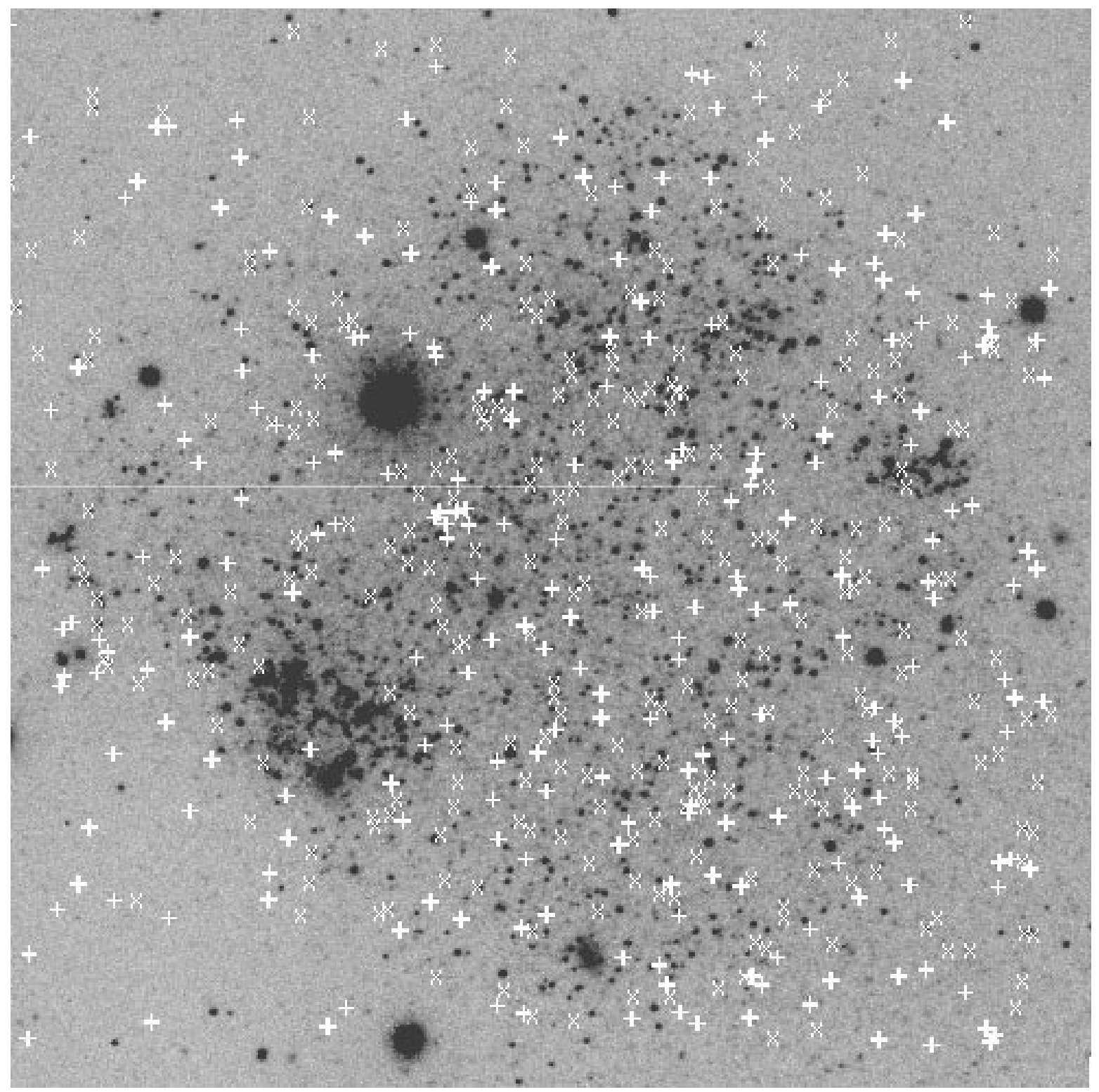

FIG. 8.-On the $V$-band image of Sextans A we show the following older populations: (1) red giants, at roughly $I \sim 22$ and $V-I \sim 1.1$, and some AGB stars, with ages between $\sim 100$ and $\sim 600 \mathrm{Myr}$ (crosses); and (2) those red giants and AGB stars with ages $\gtrsim 600$ Myr (the older red tangle and red-tail stars with ages possibly up to $\sim 3 \mathrm{Gyr} ;$ plus signs).

their distribution as a function of age are lost. Nonetheless, the distribution of this population illustrates the advanced stage of dissolution of parent OB associations. Regions in which these stars are missing are noticeable in the figure. Specifically, relatively fewer of the He-burning stars with ages greater than $50 \mathrm{Myr}$ are found at the sites of the most recent star formation (the bright blue clusters) to the east and west. In fact, no such stars are seen in the cluster to the west, again, indicating that this region is very young. In addition, the older He-burning stars near the large cluster of young stars in the southeast tend to be to the southwest edge of the cluster, indicating a possible age gradient in this large star complex.

The He-burning stars appear to mostly extend from the far north down to the far south in the galaxy, with apparent clustering toward the galaxy's optical center. Some of these stars appear along a putative bar (see, e.g., Ables 1971; A87), although they are clearly not exclusive to that region, if it exists. (Ables 1971 describes this barlike feature as being seen in the isophotes with position angle 141.5; Skillman et al. 1988 describe a possible $\mathrm{H}$ I bar with position angle $105^{\circ}$.) The integrated color of the bar feature, which appears particularly in the isophotal photometry, is $B-V=0.22$, excluding the star complex in the southeast (Ables 1971) and, thus, should be a mix of both blue and red stars. These evolved He-burning stars should therefore comprise the brighter populations along the bar, although we do not consider the optical bar structure to be entirely obvious from the distribution of these populations in Figure 7. A few of the red He-burning stars are also visible beyond the main optical galaxy; although their colors and magnitudes are quite consistent with those of other similar stars within the galaxy, these could well be foreground stars. In summary, the distribution of these older stars is clearly different from that of the younger stars.

Finally, in Figure 8 we once again show the $V$ image with two older populations: 1 ) RGB stars at roughly $I \sim 22$ and $V-I \sim 1.1$ and some AGB stars in the red tangle with ages 
between $\sim 100$ and $\sim 600 \mathrm{Myr}$ (crosses); and 2) those RGB stars and AGB stars with ages $\gtrsim 600 \mathrm{Myr}$ (the older red tangle and red-tail stars with ages possibly up to $\sim 3 \mathrm{Gyr}$; pluses). We chose the age ranges of 100-600 Myr and greater than $600 \mathrm{Myr}$ because Dohm-Palmer et al. (1997b) have found that the star formation rate between about 100$600 \mathrm{Myr}$ ago was a factor of $\sim 6$ higher than the timeaveraged rate, likely declining for ages older than this range; this is illustrated in their Figure 14.

No particular pattern or structure is obvious in the spatial distribution for the RGB stars. However, few of these stars are seen in or near the youngest star formation regions. Again, we are using a large age bin to increase our statistics, and therefore we cannot describe small timescale variations in the distribution. These stars have likely dispersed from their birthplaces over an area of diameter $\sim 5^{\prime}$. Note the approximate similarity of the distribution of RGB stars that we find and that found by Dohm-Palmer et al. (1997b; their Fig. 16) for their limited field of view, especially the possible clustering of RGB stars just east of the center.

One interesting aspect of the distributions for these stars is that they appear to also be found outside $\mu_{B} \simeq 25.9 \mathrm{mag}$ $\operatorname{arcsec}^{-2}$. Again, based on Table 2, one might suspect that $\sim 15 \%-30 \%$ of these stars are merely in the foreground. But, again, the colors and magnitudes for the stars found outside the main body of the galaxy are consistent with those red giants well within the galaxy, and a large fraction therefore could be members of Sextans A. These stars may comprise a more extended halolike structure, which is also found for a number of dwarf irregular galaxies (e.g., Minniti \& Zijlstra 1996).

The older RGB and AGB stars with ages $\gtrsim 600 \mathrm{Myr}$ are also uniformly distributed across and beyond the main body of the optical galaxy, with no apparent clustering or structure. Again, the stars seen beyond $\mu_{B} \simeq 25.9 \mathrm{mag}$ $\operatorname{arcsec}^{-2}$ have colors and magnitudes consistent with those within the galaxy, but probably up to about one-third of these stars could be in the foreground. The quite homogeneous distribution is what one would expect for an older halo field population. Hunter \& Plummer (1996) point out that star formation in Sextans A must once have occurred further out in the galaxy than is seen today, given the size of the Holmberg radius. They also point out that in the southwest they can trace faint stars as far out as $1.5 \mathrm{kpc}$ from the center.

What we find for stars older than $100 \mathrm{Myr}$ is consistent with the picture developed by Dohm-Palmer et al. (1997b, 1998), in which star formation, at a significantly lower rate than the current rate, percolates in coherent regions and propagates to other neighboring regions within their field of study during the time range of $\sim 100-600 \mathrm{Myr}$. The rate is likely lower for ages older than $600 \mathrm{Myr}$, but the pattern and propagation of star formation appear from our data to have been similar in nature. In particular, Dohm-Palmer et al. (1998) show that from 300-400 Myr, star formation migrated from the northeast to the center, peaking there around $350 \mathrm{Myr}$ ago. Our results seem to imply that this centrally concentrated star formation continued to peak from $\lesssim 100 \mathrm{Myr}$.

\section{RELATIONSHIP OF THE STARS TO THE GAS}

We now examine the spatial distribution of the various stellar populations to that of the gas in Sextans A. We can consider only the ionized and neutral components of the gas in the galaxy, since molecular gas has not been detected (Ohta et al. 1993 found only a low upper limit for the luminosity of $\mathrm{CO}$ ).

\subsection{Ionized Hydrogen}

First, we examine the distribution of blue stars relative to the ionized gas, as seen from our $\mathrm{H} \alpha+[\mathrm{N}$ II $]$ image. In Figure 9, we show our $\mathrm{H} \alpha$ map, which is qualitatively similar to that in Aparicio \& Rodriguez-Ulloa (1992) and Hodge, Kennicutt, \& Strobel (1994a). All the $\mathrm{H} \alpha$ emission appears to be nonstellar. The $\mathrm{H}$ II regions are distributed in two arcs separated by nearly $4^{\prime}$ (with the brightest patches of emission in the southeast). Fainter emission can also be seen. Our $\mathrm{H} \alpha$ image, although continuum-subtracted, is not flux-calibrated, but comparing our map with that in Hodge et al. (1994a), we estimate that our detection limit for H II regions is $\sim 2.5 \times 10^{-14} \mathrm{ergs} \mathrm{cm}^{-2} \mathrm{~s}^{-1}$, which, at a distance of $1.4 \mathrm{Mpc}$, corresponds to a luminosity of $\sim 6 \times 10^{36} \mathrm{ergs}$ $\mathrm{s}^{-1}$. Some of the fainter structures seen on the deep $\mathrm{H} \alpha$ map in Hunter \& Gallagher (1997), such as the long filaments (e.g., their filaments 1 and 2) and fainter shells (e.g., their shell 4) are just barely visible on our map and are somewhat below our detection limit.

In Figure 9, we show the blue main-sequence stars (as in Fig. 6 , with $-1.2 \lesssim U-B \lesssim-1.0$ and $V \lesssim 22$ ) relative to the $\mathrm{H} \alpha$ emission. These stars are roughly spectral type B0 and hotter, and $\lesssim 8-10 \mathrm{Myr}$ old (at the assumed metallicity of Sextans A, this corresponds to a main-sequence turnoff mass of $\sim 20 M_{\odot}$ ). Stars cooler than this do not contribute significantly to the UV continuum flux that can produce $\mathrm{H}$ II regions in the galaxy. One can see that the hot, massive stars, as expected, are well correlated with the ionized gas, both bright and faint (in fact, a cluster of six stars cannot be distinguished from the dark contrast in Fig. 9 for the $\mathrm{H}$ II region "No. 17," following the Hodge et al. numbering scheme, or "No. 7," following that of Aparicio \& Rodriguez-Ulloa 1992). Thus, we have accounted for the ionizing sources for the majority of the $\mathrm{H}$ iI gas.

A smaller number of presumed OB stars are not associated with any $\mathrm{H}$ II regions above the detection limit of our study. This is true also for the regions detected by Aparicio \& Rodriguez-Ulloa (1992) and Hodge et al. (1994a), although some appear to be associated with some of the fainter emission seen in the Hunter \& Gallagher (1997) map. In addition, some of the fainter emission features, based on closer inspection of our images, may be ionized by unresolved small clusters of hot stars, as is also likely the case for the bright knotty emission region, which is Hodge et al. (1994a) H II region No. 20 and Aparicio \& Rodriguez-Ulloa (1992) No. 2. However, the inner area of the galaxy is relatively devoid of massive stars and $\mathrm{H}$ II regions (the inner portion of the galaxy may have several faint filaments of emission, and, as Hunter \& Gallagher 1997 point out, these filaments might comprise a larger shell structure associated with the bright $\mathrm{H}$ II complex in the southeast). The bulk of the massive star formation within the last $\sim 20 \mathrm{Myr}$ has been spatially and temporally correlated along the edge of the main surface brightness for the galaxy.

\subsection{Neutral Hydrogen}

Next, we investigate the relationship of the stars to the neutral gas. We have utilized a radio $21 \mathrm{~cm} \mathrm{H}$ I map from Graham \& Westpfahl (1998). Here we dispense with the 


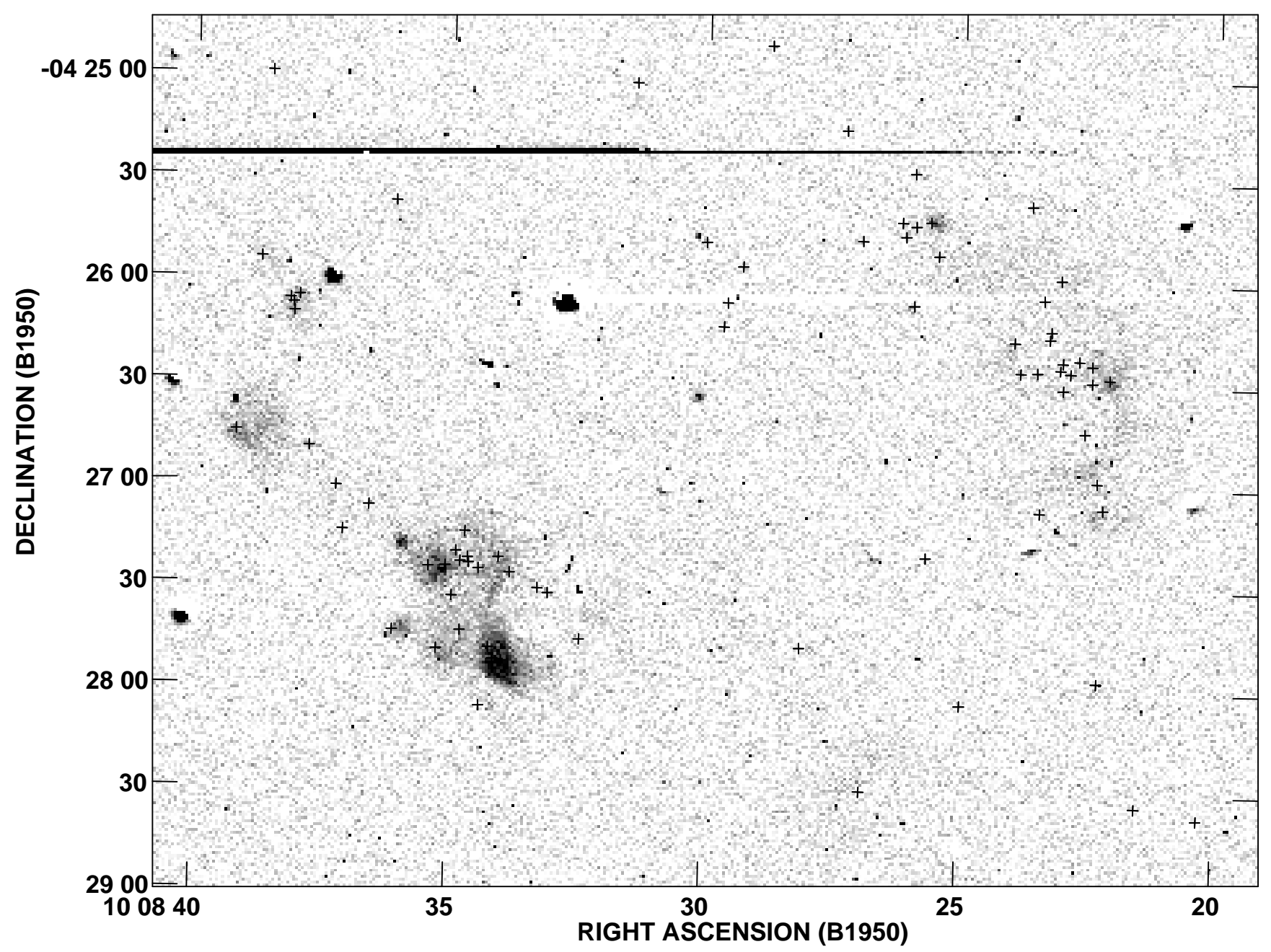

FIG. 9.-Distribution of stars in Sextans A with $-1.2 \lesssim U-B \lesssim-1.0$ and $V \lesssim 22$ relative to the ionized hydrogen (H $\alpha+[\mathrm{N}$ II $]$ emission)

details of their $\mathrm{H}$ I data and only note that this $\mathrm{H}$ I map was made using the Very Large Array (VLA) of the National Radio Astronomy Observatory ${ }^{4}$ at the redshifted frequency of the $21 \mathrm{~cm}$ line of neutral hydrogen appropriate for the galaxy in the C-configuration in 1992 May and the Dconfiguration in 1992 July. (B-configuration observations were also made in 1994 June but are not included in the map shown here.) These two sets of data were then combined, resulting in a naturally weighted map with resolution $34^{\prime \prime} \times 23^{\prime \prime}$ and rms noise in the channel maps of $1.36 \mathrm{mJy}$ beam $^{-1}$. Both the resolution and the sensitivity are higher than those for the H I map presented in Skillman et al. (1988). In addition, with the short spacings sampled in the most compact configuration, the maps are sensitive to more extended emission than were the Skillman et al. data. ${ }^{5}$

In Figure 10, we show the total $\mathrm{H}$ I column density map for Sextans A. What Puche \& Westpfahl (1994) consider a central depression, or "hole," within an H I shell is clearly

\footnotetext{
4 The National Radio Astronomy Observatory is a facility of the National Science Foundation operated under cooperative agreement by Associated Universities, Inc.

${ }^{5}$ An initial analysis of the data by D. Puche, including moment maps, is available on the World Wide Web at http://cfa-www.harvard.edu/ projects/nga/sexa.html.
}

visible; Skillman et al. (1988) describe the H I morphology as a "horseshoe or ringlike" structure around a central minimum with a sharp outer boundary. This hole, $\sim 1.5 \mathrm{kpc}$ in diameter, encompasses nearly the entire main optical surface brightness of the galaxy. The highest column density forms the two large spurlike or arclike structures toward the southeast (peak column, $\sim 1.6 \times 10^{22} \mathrm{~cm}^{-2}$ ) and the northwest (peak column, $\sim 1.2 \times 10^{22} \mathrm{~cm}^{-2}$ ), as also seen in the map by Skillman et al. (1988) and highlights the nearly complete ring or shell. The column density in the center of the galaxy in the hole is $\sim 1.2 \times 10^{21} \mathrm{~cm}^{-2}$. The fine detail in this map indicates substructuring of smaller, fainter clumps, or clouds, and holes within the larger $\mathrm{H}$ I hole as well. In addition, one can see that $\mathrm{H}$ I extends well beyond the $\mathrm{H}$ I ring or shell, as well as the main optical galaxy, consistent with other normal galaxies and what van Zee, Haynes, \& Giovanelli (1995) found for their sample of dwarf galaxies. A fascinating feature of the map is the "hook" of gas extending from the disk to the west. Again, we defer here to Graham \& Westpfahl (1998) for a complete presentation of these $\mathrm{H}$ I data.

In Figure 10 we also show the positions of the same blue stars as in Figure 9 (pluses). What should be kept in mind is that the gas disk is likely thicker than that for spirals (Puche et al. 1992) and that the $\mathrm{H}$ I shell is roughly spherical. What 


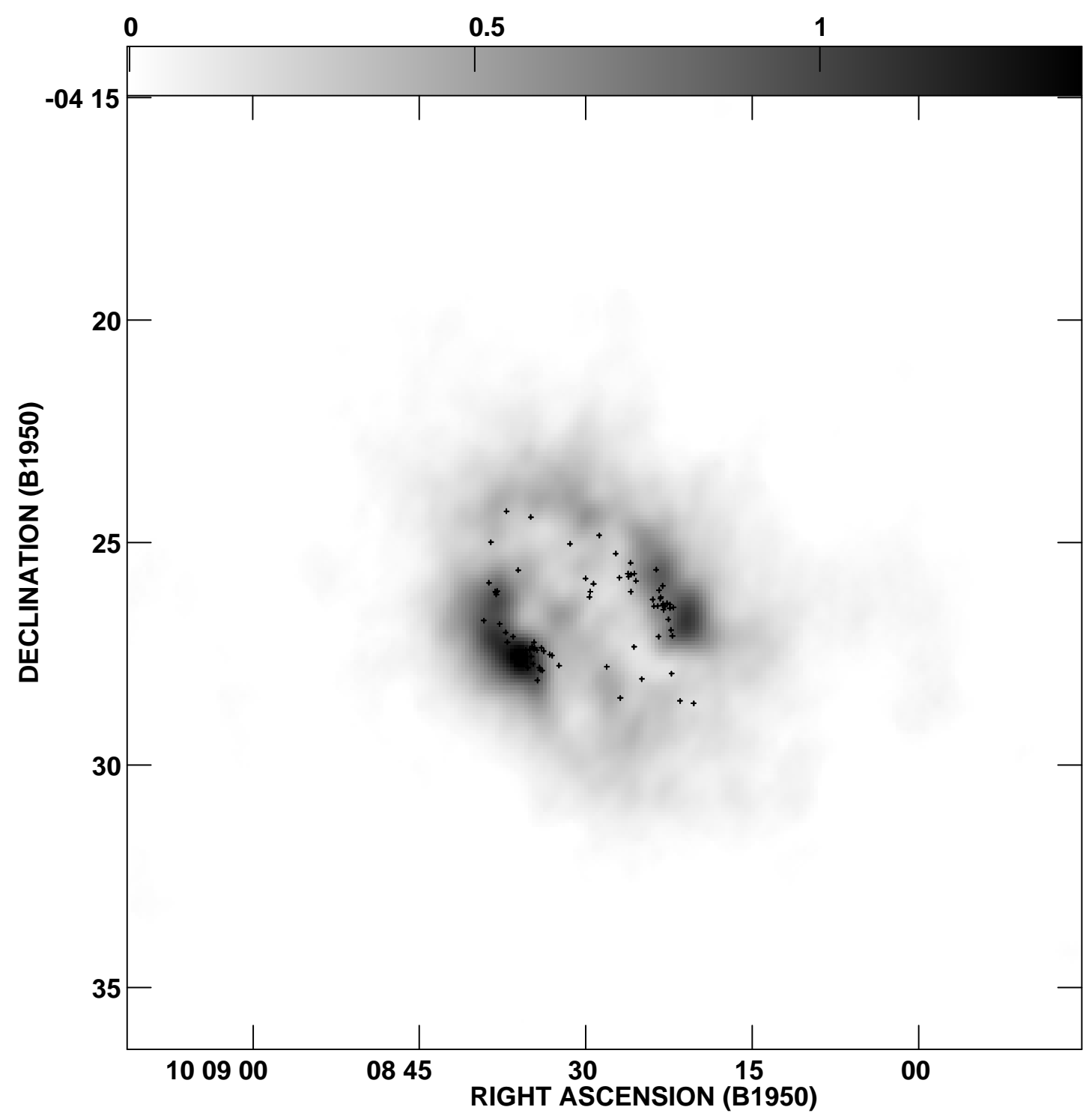

FIG. 10.-Distribution of the stars in Fig. 9 relative to neutral hydrogen $(\mathrm{H} \mathrm{I})$ as shown from a column density map derived from $21 \mathrm{~cm}$ VLA observations in Graham \& Westpfahl (1998). The color bar gives the approximate H I column density in $10^{22} \mathrm{~cm}^{-2}$.

is most striking is that the bluest, youngest stars are found preferentially and predominantly along the inner edge of the large $\mathrm{H}$ I hole, particularly along the inner edge of, and not coincident with, the two large complexes of $\mathrm{H}$ I gas to the southeast and the northwest. Far fewer of these stars are seen within or outside of this locus.

In Figure 11 one can also see, not unexpectedly, that the $\mathrm{H} \alpha$ emission is along the inner edges of the $\mathrm{H}$ I hole. The two large $\mathrm{H}$ II region complexes, in the southeast and the northwest, are not coincident with the brightest $\mathrm{H}$ I peaks, but, in fact, straddle the inner edge of both peaks. Hunter \& Plummer (1996) noted that the distribution of $\mathrm{H} \alpha$ does not extend as far as the $\mathrm{H}$ I gas distribution. This is consistent with the fact that the most recent star formation is occurring primarily along the inner edge of the $\mathrm{H}$ I hole.

In Figure 12, we show the distributions of the young, blue, main-sequence stars, supergiants, and He-burning stars together, as in Figure 6. One can see that the overall spatial distribution of stars with ages up to $\sim 50 \mathrm{Myr}$ appears to have a shape and symmetry axis similar to the current distribution of the $\mathrm{H}$ I gas and to the youngest stars but with a smaller radius. If the peak velocity dispersion in the H I ( 9-10 $\mathrm{km} \mathrm{s}^{-1}$; Skillman et al. 1988; Graham \& Westpfahl 1998) represents a possible expansion of the gas outward to form the large $\mathrm{H}$ I hole, then to produce the current size of the hole $(\sim 1.5 \mathrm{kpc})$ would take $\sim 80 \mathrm{Myr}$, assuming a constant expansion rate. If stars have been consistently forming along the inner edge of the $\mathrm{H}$ i hole as the gas homologously expanded, then we might expect stars with ages up to $\sim 50 \mathrm{Myr}$ to share a similarly ring-shaped spatial distribution as the most recently formed stars, albeit closer to the galaxy's center, since $\sim 50 \mathrm{Myr}$ ago the $\mathrm{H} \mathrm{I}$ hole would have been $\sim 0.5 \mathrm{kpc}$ smaller in radius. (However, OB associations would have dispersed somewhat over a $\sim 50$ Myr timescale.)

In Figure 13 we show the distributions of the older Heburning stars, as in Figure 7, with ages $\sim 50-100$ Myr. What is immediately evident is that these older stars are concentrated more toward the center of the $\mathrm{H}$ i hole and bear relatively little relationship with the $\mathrm{H} \mathrm{I}$ hole, shell, and substructuring than do the younger stars in Figure 12. We point out that regions within the $\mathrm{H}$ I hole exist where no 


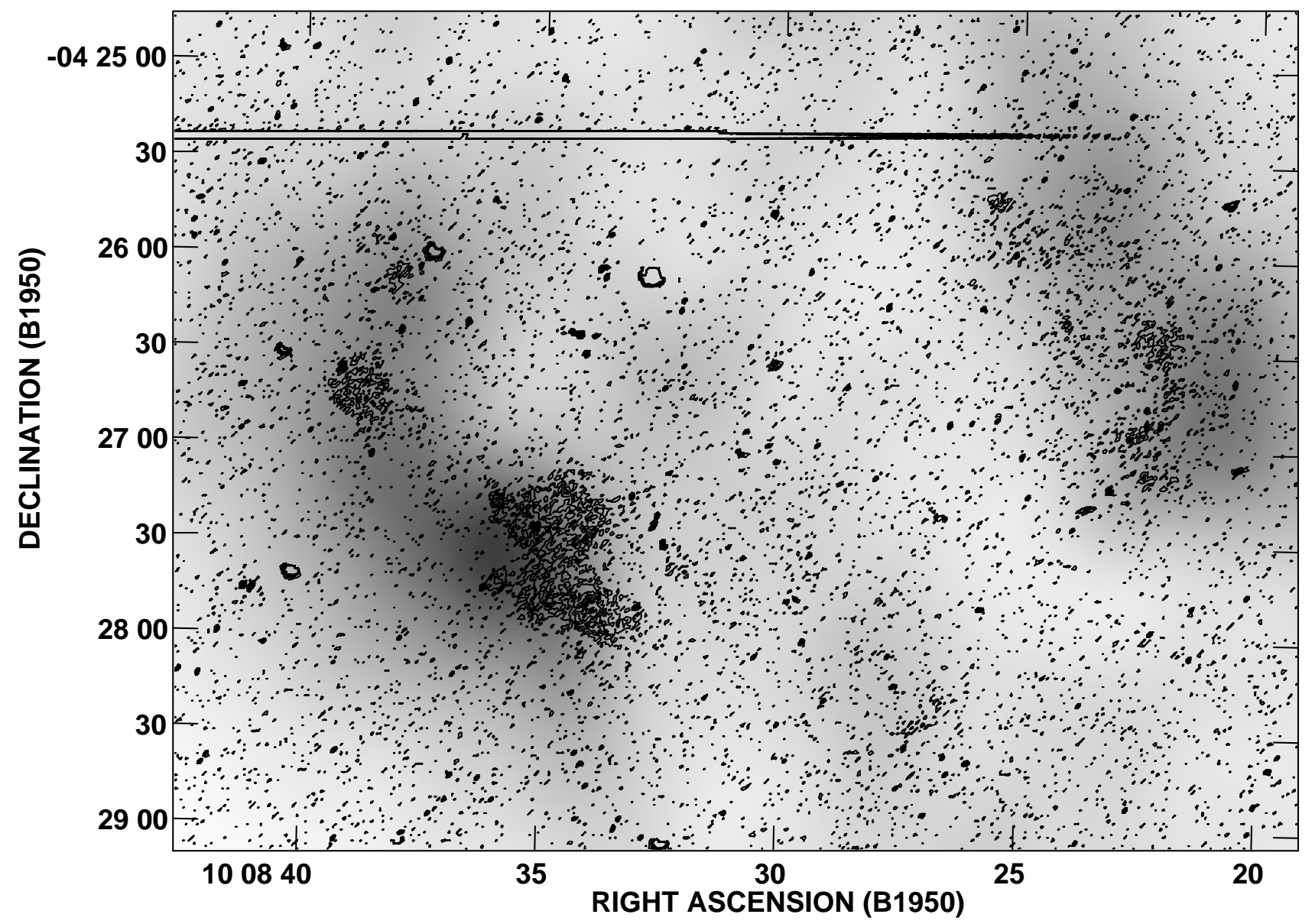

FIG. 11.-Distribution of ionized hydrogen ( $\mathrm{H} \alpha+[\mathrm{N} \mathrm{II}]$ emission) in Sextans A (shown in Fig. 9) relative to the $\mathrm{H}$ I (shown in Fig. 10)

stars from these older generations appear to have formed. It is clear that the gas must have been more centrally concentrated in the past than it is now.

Finally, in Figure 14 we show the distributions of the older RGB and AGB stars, as in Figure 8, with ages from $\sim 100 \mathrm{Myr}$ to $1 \mathrm{Gyr}$ or so. Here we see no relationship between these stars and the current structure of the neutral gas, consistent with the substantial dispersal of these stars from their birthplaces. The populations of older stars appear nearly uniformly distributed relative to the observed $\mathrm{H}$ I. Although our optically imaged field encompasses nearly the entire $\mathrm{H}$ I hole, it does not overlap much with the gas beyond the $\mathrm{H}$ I shell. The indications in Figure 14, however, are that stars had formed long ago well beyond the current $\mathrm{H}$ I shell structure. The $\mathrm{H}$ I gas in the extended disk outside the shell implies that the star-forming molecular gas may well have existed (and may still exist) there.

\section{DISCUSSION}

What could have been responsible for the substantial increase in the rate and spatial concentration of the recent star formation in Sextans A? The answer to this puzzle has ramifications for our understanding of both this particular galaxy and the bursts of star formation occurring in other dwarf irregular galaxies. Dohm-Palmer et al. (1997b) conclude that sometime within the last $100 \mathrm{Myr}$ or less, an event or events transpired that launched the galaxy into its present star-forming high state (although the movie in Dohm-Palmer et al. 1998 might imply that this event occurred as early as $\sim 350 \mathrm{Myr}$ ago). We briefly speculate here on some of the possible mechanisms. A tidal interaction with nearby Sextans B has been dismissed above as a possibility because of the large separations between the two galaxies.

One possibility is the presence of a bar potential in the galaxy. Several authors have stressed the existence of a possible barlike feature, evident both in the optical isophotal data (Ables 1971) and from the gas kinematics (Skillman et al. 1988). A misalignment of the $\mathrm{H}$ I morphological axis with the kinematical axis and, possibly, with the optical morphology, is typically an indication of a bar. The bar feature appears somewhat off center, as is true for Magellanic irregular galaxies more massive than Sextans A. The H I bar presumably connects the two pronounced maxima in the $\mathrm{H}$ I distribution and may be associated with the gas pileup at its ends, where the most prominent recent star formation is occurring.

Although less is known about bars in dwarf irregular galaxies than for other larger galaxies, we can make inferences based on what is known for the more massive systems. Bars in irregular galaxies (generally of greater mass than Sextans A) are common and may possibly arise from interaction with small neighboring $\mathrm{H}$ I clouds (Wilcots, Lehman, \& Miller 1996). A bar can form in a disk in about one-half to one rotation period as a result of a merger or tidal interaction (Noguchi 1987; Barnes \& Hernquist 1991). In addition, late-type galaxies tend to have $\mathrm{H}$ I gas-rich bars (Hunter \& Gottesman 1996) with star formation occurring 


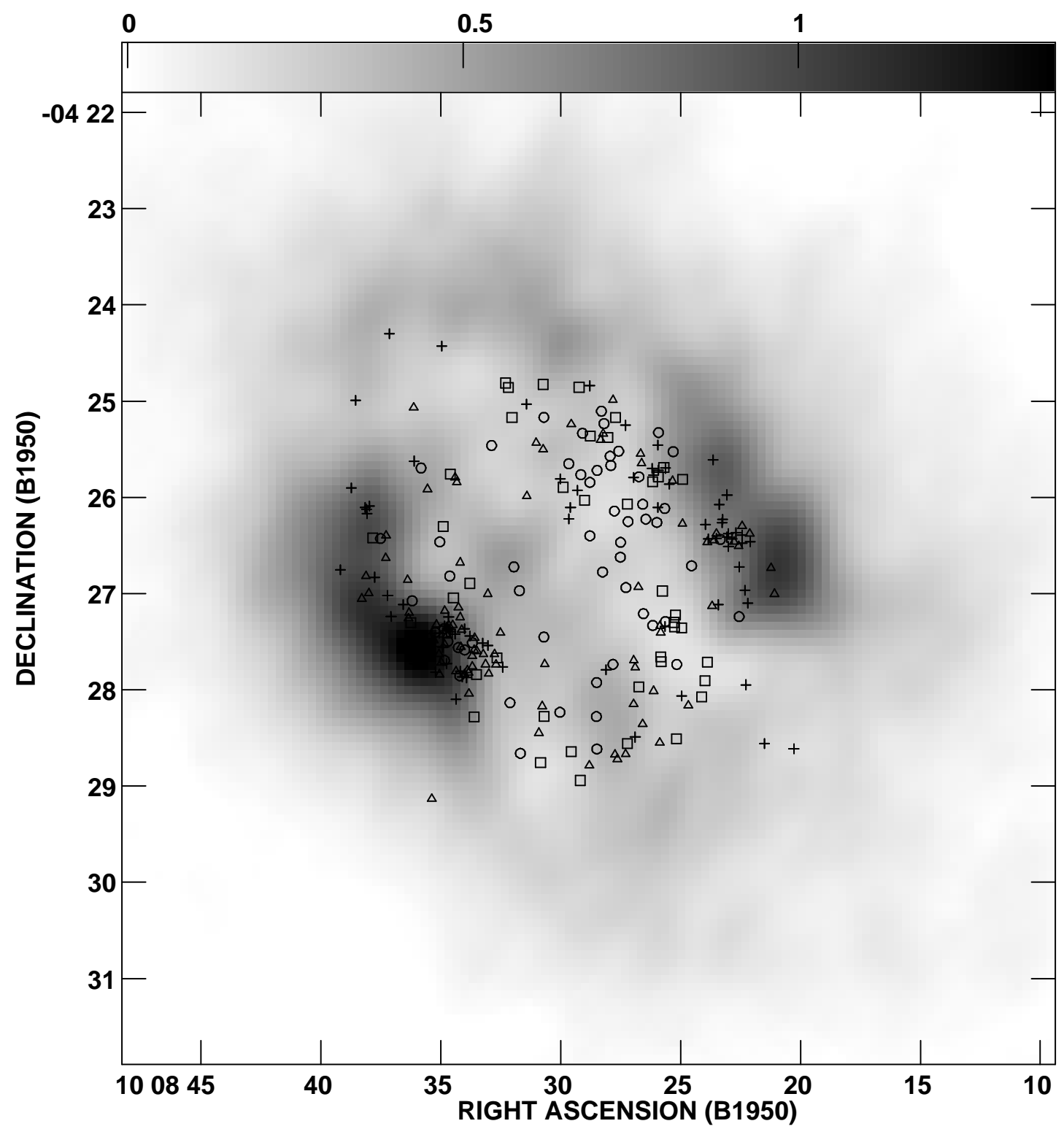

FIG. 12.-Distribution of the stars in Fig. 6 relative to neutral hydrogen $(\mathrm{H} \mathrm{I})$. The symbols are the same as in Fig. 6, except that the likely main-sequence turnoff stars and supergiants are represented with triangles, rather than crosses. The color bar is the same as in Fig. 10.

along the bar (Phillips 1996), which appears to be the trend for the Magellanic irregular galaxies NGC 4618 (Odewahn 1991) and NGC 4449 (Hunter \& Thronson 1996), for example.

The main difficulties with a bar explanation for Sextans A are the distribution of gas and star formation in the galaxy and the necessary timescale for bar evolution. Induced inflow of gas along a bar (e.g., Schwarz 1984) could account for the fact that the gas in Sextans A was once more centrally concentrated $\gtrsim 50 \mathrm{Myr}$ ago. However, the $\mathrm{H}$ I gas and star formation is currently concentrated in a shell around a central hole. The evolution of a bar, in the absence of interaction, likely requires several rotation periods. However, a bar can be destroyed in about one rotation period with the formation of a long-lived outer gaseous ring after a tidal interaction or a central accumulation of mass (Athanassoula 1996). Redistribution of the gas from the bar into a ring could explain the current gas distribution. Yet, the dynamical timescale for Sextans A is longer than these model bar evolutionary timescales, since the galaxy has a rotation period $\sim 400 \sin i \mathrm{Myr}$ (Skillman et al. 1988).

Furthermore, we have isolated the region of the galaxy thought to be the putative optical bar (cf. Ables 1971) and find that the ages of the bar stars are typically $\gtrsim 40-50 \mathrm{Myr}$. We find no difference, then, between the ages of these stars and those of the stars generally throughout the central regions of the galaxy. The presence of an optical bar is therefore not convincing; we may merely be seeing the remnants of an extended OB association or associations analogous to Shapley constellation III and environs in the LMC.

A second possibility is the effect of a merger or accretion event in the past $\sim 100 \mathrm{Myr}$ (with or without bar formation). This is the mechanism for Sextans A that is supported in Graham \& Westpfahl (1998). Evidence for merger events appears to exist for a number of dwarf galaxies. Saio, Kato, \& Nomoto (1992) propose such a model 


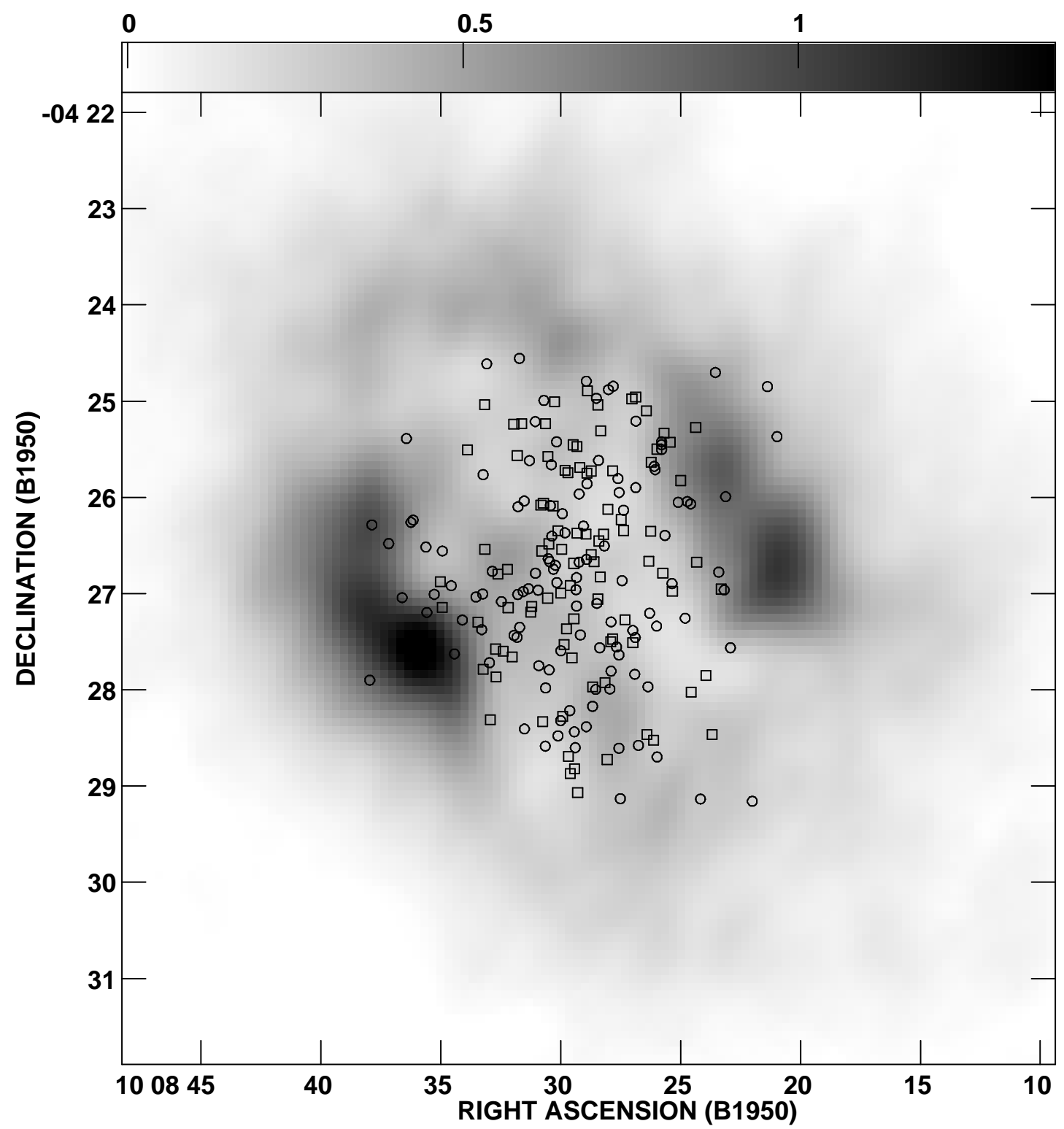

Fig. 13.-Distribution of those stars in Fig. 7 relative to neutral hydrogen $(\mathrm{H} \mathrm{I})$. The symbols are the same as in Fig. 7 . The color bar is the same as in Fig. 10.

for IC 10. Of particular curiosity in the case of Sextans A is the $\mathrm{H}$ I gas extending westward from the $\mathrm{H}$ I disk. Graham $\&$ Westpfahl claim that the velocity field for the galaxy can be explained as the result of a merger. Mergers are also known to result in a central concentration of the molecular gas in galaxies. Thus, a merger model cannot be easily dismissed. However, it alone does not offer an explanation for the current distribution of star formation in Sextans A.

A third possibility is the mechanism of supernova-driven expansion and sequential star formation, at least for more recent epochs. Hunter et al. (1998) have recently suggested that sequentially triggered star formation driven by mechanical energy input by concentrations of massive stars is likely to be an important mechanism for cloud formation in irregular galaxies. The fact that the current star formation in Sextans A is occurring mostly on the edge of the $\mathrm{H}$ I hole is consistent with cold gas pileup and compression at this locus. This would occur if the gas within the $\mathrm{H}$ I hole is expanding outward into the extended $\mathrm{H}$ I envelope seen in the radio data. The behavior of the current star formation is remarkably similar to the star formation occurring particularly along the largest $\mathrm{H}$ I holes seen in Ho II (Puche et al. 1992; see also Tongue \& Westpfahl 1995) and IC 2574 (Martimbeau et al. 1994). A local example may also be constellation III and LMC 4 in the LMC (Dopita, Mathewson, \& Ford 1985; Grebel \& Brandner 1998).

As massive stellar populations have formed and evolved in the past in the interior of Sextans A, they have input significant energy into the ISM through stellar winds (e.g., Leitherer et al. 1992) and ended their lives as supernovae. The resulting hot gas expands outward from the interior of the galaxy, pushing on the colder gas, compressing it, forming clouds, and triggering further star formation as the galaxy evolves.

Is this mechanism plausible for Sextans A, given the kinematics of the gas? As discussed in $\S 5.2$, if the peak velocity dispersion in the H I ( 9-10 $\mathrm{km} \mathrm{s}^{-1}$; Skillman et al. 1988; Graham \& Westpfahl 1998) represents a possible expansion 


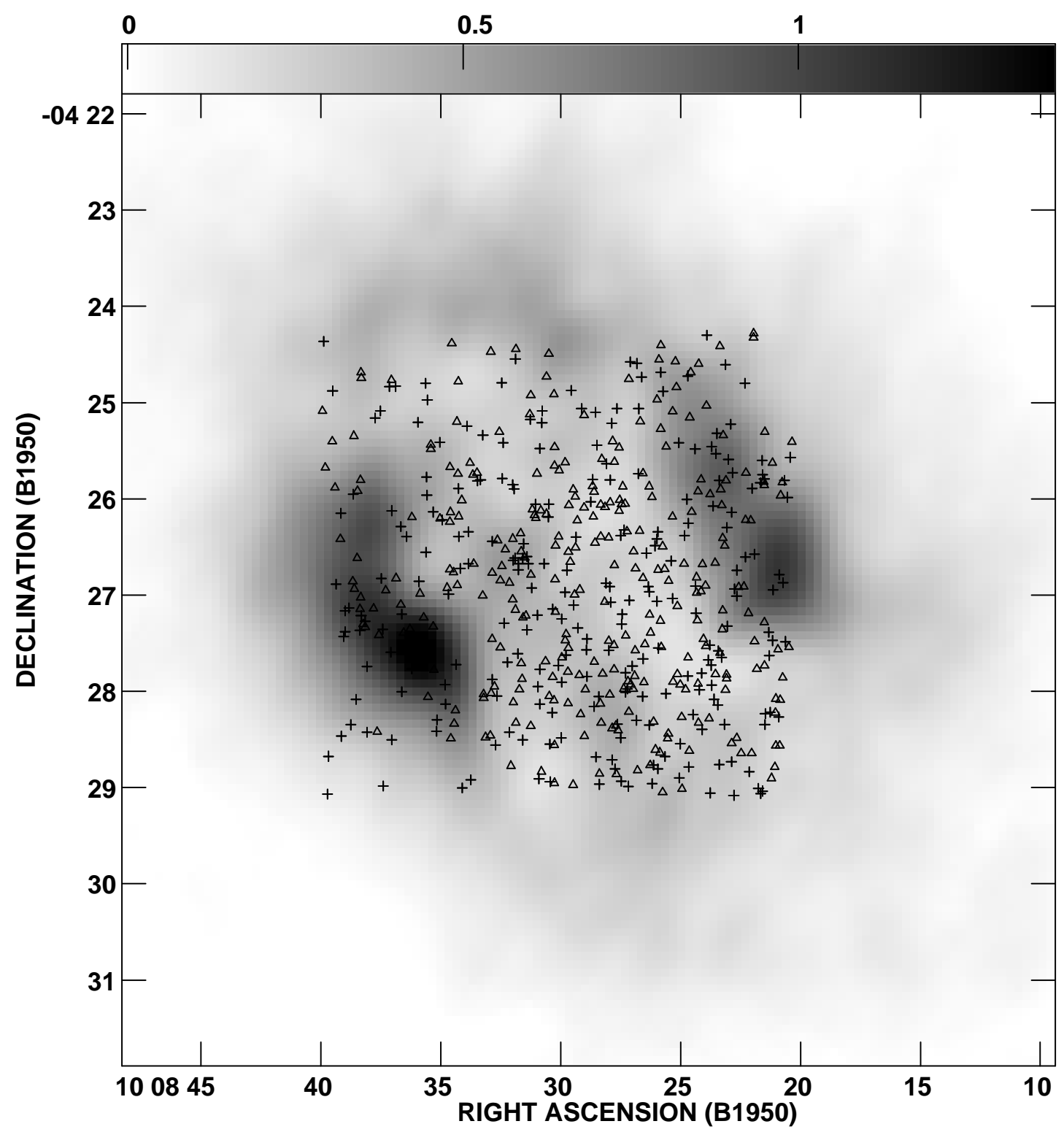

FIG. 14.-Distribution of those stars in Fig. 8 relative to neutral hydrogen $(\mathrm{H} \mathrm{I})$. The symbols are the same as in Fig. 8, except that the $\sim 100-\sim 600 \mathrm{Myr}$ RGB and AGB stars are represented with triangles rather than crosses. The color bar is as in Fig. 10.

of the gas to form the large $\mathrm{H}$ i hole, then the age of the hole would be $\sim 80$ Myr. This timescale is consistent with the fact that the star formation rate substantially increased in the last $100 \mathrm{Myr}$ and also agrees with the ages of stars near the center of Sextans A, which would presumably be the remaining less massive siblings of the supernova progenitors from those stellar generations. Furthermore, the distributions of populations with ages less than $50 \mathrm{Myr}$ is suggestive of a progression from oldest stars inward to the youngest outward within the $\mathrm{H}$ I hole, consistent with an expanding front of star formation outward from the center.

A major difficulty with this scenario is that, although Puche \& Westpfahl (1994) claim evidence for expanding gas, Graham \& Westpfahl (1998), from the very same data set, do not find convincing evidence for an expanding shell, i.e., characteristic double-peaked velocity profiles, particularly at the velocity dispersion peak, which they find near the galaxy's center in the lowest column density region. However, it may be difficult to see such a feature in these low brightness regions. Furthermore, Domgoergen, Bomans, \& de Boer (1995) and Points et al. (1998) found an absence of expansion for LMC 4 and LMC 2, respectively.

Another potential difficulty is that Rhode, Salzer, \& Westpfahl (1998), from deep optical CCD imaging, recently claim not to find remnant populations at the centers of $\mathrm{H} \mathrm{I}$ holes in Ho II and other dwarfs. However, Rhode et al. searched for remnant main-sequence populations at the hole centers, whereas it is clear from our CMDs that the remnant populations would be most readily identified as evolved He-burning stars, which are $\sim 2$ mag brighter, and significantly redder, than main-sequence stars of the same age. (Interestingly, red supergiants might dominate the light from a remnant cluster, since in systems with the low metallicities of dwarf irregular galaxies these stars may not necessarily be the progenitors of all massive supernovae, as we have directly witnessed with SN 1987A in the LMC; see Woosley 1988; Saio et al. 1988.) For Sextans A, we believe that our imaging shows several populations of a feasible age 
near the center of the large hole, which are suitable candidates for remnant sibling stars to the supernovae progenitors.

Of course, some combination of these various mechanisms may have been at work during the galaxy's history. It is possible that the galaxy has experienced the effects of a merger or accretion event, or of a bar potential sometime in the past, resulting in a significant increase in the star formation rate. The increased massive star formation resulted in increased feedback to the ISM, which may have resulted in an expanding bubble of hot gas, which has promoted the current star formation we are witnessing. We simply do not have enough information.

The trigger for the current burst of star formation in Sextans A remains a mystery, although we have shed additional new light through our optical imaging of the galaxy and our comparison with the gas components. DohmPalmer et al. (1997a, 1997b) have also provided some vital clues, but because of the limited field size of the HST WFPC2 camera, they sampled only about one-half of the galaxy, and therefore, their results are only applicable to this smaller field. Since star formation has occurred in bursts in various regions of Sextans A, sampling a larger area of the galaxy provides a broader view of the recent star formation history.

Unfortunately, one important additional clue that is missing is the distribution of the molecular gas, about which we have no information other than the low upper limit to the luminosity of the CO tracer (Ohta et al. 1993). We do not know the mass or the extent of the actual gaseous raw material for star formation in the galaxy. That information would help resolve questions about the nature and history of star formation in Sextans A.

\section{CONCLUSIONS}

From our ground-based observations of the Local Group dwarf irregular galaxy Sextans $A$ in $U B V(R I)_{\mathrm{C}}$ and $\mathrm{H} \alpha$, we have found that current star formation is occurring mostly along the inner edge of the large $\mathrm{H}$ i shell surrounding a hole, or depression, in the $\mathrm{H}$ I column density, consistent with cold gas pileup and compression of the gas at this locus. The distribution of stars with ages $\lesssim 50 \mathrm{Myr}$ indicates an outward progression of star formation with time. Regions exist where the star formation appears to be exclusively recent and where no star formation has occurred within the last $50 \mathrm{Myr}$. Older star formation, from $\sim 50$ $100 \mathrm{Myr}$, appears to have some spatially coherent structure and is more centrally concentrated than the most recent star formation. The oldest star formation that we can accurately sample appears to be uniformly distributed across Sextans A, even beyond a surface brightness of $\mu_{B} \simeq 25.9 \mathrm{mag}$ $\operatorname{arcsec}^{-2}$ or a radius $r \simeq 2^{\prime} .3$.

The recent bursts in star formation are not only coherent in time but also in space across Sextans A. The trigger of these bursts remains unknown; however, we believe we have presented the strongest evidence so far that supernova-driven expansion of the gas may be responsible for at least some of the star formation over the galaxy's history. The expansion of the gas due to episodic sequential star formation within the galaxy is a direct trigger for further star formation as the galaxy evolves. We note that the expected expansion age of the $\mathrm{H}$ I shell is $\sim 80 \mathrm{Myr}$ (assuming an expansion velocity of $\sim 10 \mathrm{~km} \mathrm{~s}^{-1}$ and a shell diameter of $\sim 1.5 \mathrm{kpc}$ ), which is consistent with the ages of the older, centrally concentrated evolved stars, which may be the remnants of the populations that included the massive stars responsible for the expansion.

We are grateful to Ralph Young Shuping for his photometric calibration observations of Sextans A at the KPNO $0.9 \mathrm{~m}$ telescope, to NOAO director Sydney Wolff for scheduling these observations, and Dave Summers for his assistance at the KPNO $2.1 \mathrm{~m}$ telescope. We thank Maggie Graham and Dave Westpfahl for making their total H I map available to us. S. V. D. is grateful for assistance provided by Alex Filippenko and also to the UCLA Division of Astronomy and Astrophysics. We thank Jean Turner, Tammy Smecker-Hane, Dave Westpfahl, and Dave Meier for helpful discussions. We also thank the referee for very helpful comments and suggestions. This research was supported by a grant from NASA administered by the American Astronomical Society.
Ables, H. 1971, Publ. US Naval Obs., 2d Ser., 20, Part 4

Aparicio, A., \& Gallart, C. 1995, AJ, 110, 2105

Aparicio, A., Gallart, C., \& Bertelli, G. 1997, AJ, 114, 669

Aparicio, A., Gallart, C., Chiosi, C., \& Bertelli, G. 1996, ApJ, 469, L97

Aparicio, A., Garcia-Pelayo, J. M., Moles, M., \& Melnick, J. 1987, A\&AS, 71, 297 (A87)

Aparicio, A., \& Rodriguez-Ulloa, J. A. 1992, A\&A, 260, 77

Athanassoula, E. 1996, in ASP Conf. Ser. 91, Barred Galaxies, ed. R. Buta, D. A. Crocker, \& B. G. Elmegreen (San Francisco: ASP), 309

Barnes, J. E., \& Hernquist, L. E. 1991, ApJ, 370, L65

Bertelli, G., Bressan, A., Chiosi, C., Fagotto, F., \& Nasi, E. 1994, A\&AS, 106,275

Braun, J. M., Bomans, D. J., Will, J.-M., \& de Boer, K. S. 1997, A\&A, 328, 167

Burstein, D., \& Heiles, C. 1984, ApJS, 54, 33

Cardelli, J. A., Clayton, G. C., \& Mathis, J. S. 1989, ApJ, 345, 245

Cash, W., Charles, P., Bowyer, S., Walter, F., Garmire, G., \& Riegler, G. 1980, ApJ, 238, L71

Chevalier, R. A. 1974, ApJ, 188, 501

Chiosi, C., Bertelli, G., \& Bressan, A. 1992, ARA\&A, 30, 235

Costa, E., \& Frogel, J. A. 1996, AJ, 112, 2607

Dohm-Palmer, R. C., Skillman, E. D., Saha, A., Tolstoy, E., Mateo, M., Gallagher, J., Hoessel, J., Chiosi, C., \& DuFour, R. J. 1997a, AJ, 114, 2514

-1997b, AJ, 114, 2527

. 1998, AJ, 115, 152

Domgoergen, H., Bomens, D. J., \& de Boer, K. S. 1995, A\&A, 296, 523

Dopita, M. A., Mathewson, D. S., \& Ford, V. L. 1985, ApJ, 297, 599

\section{REFERENCES}

Drissen, L., Roy, J.-R., \& Robert, C. 1997, ApJ, 474, L35

Freedman, W. L. 1985, ApJ, 299, 74

Gerola, H., \& Seiden, P. E. 1978, ApJ, 223, 129

Gerola, H., Seiden, P. E., \& Schulman, L. S. 1980, ApJ, 242, 517

Graham, M., \& Westpfahl, D. 1998, in preparation

Grebel, E. K., \& Brandner, W. 1998, in The Magellanic Clouds and Other Dwarf Galaxies, ed. T. Richtler \& J. M. Braun, in press

Grebel, E. K., Roberts, W. J., \& Brandner, W. 1996, A\&A, 311, 470

Greggio, L., Marconi, G., Tosi, M., \& Focardi, P. 1993, AJ, 105, 894

Heiles, C. 1979, ApJ, 229, 533

. 1984, ApJS, 55, 585

Hodge, P., Kennicutt, R. C., \& Strobel, N. 1994a, PASP, 106, 765

Hodge, P., Strobel, N. V., \& Kennicutt, R. C. 1994b, PASP, 106, 309

Hoessel, J. G., \& Melnick, J. 1980, A\&A, 84, 317

Hoessel, J. G., Schommer, R. A., \& Danielson, G. E. 1983, ApJ, 274, 577 (HSD)

Hunter, D. A. 1997, PASP, 109, 937

Hunter, D. A., Elmegreen, B. G., \& Baker, A. L. 1998, ApJ, 493, 595

Hunter, D. A., \& Gallagher, J. S. 1986, PASP, 98, 5

.1997, ApJ, 475, 65

Hunter, J. H., \& Gottesman, S. T. 1996, in ASP Conf. Ser. 91, Barred Galaxies, ed. R. Buta, D. A. Crocker, \& B. G. Elmegreen (San Francisco, ASP), 398

Hunter, D. A., \& Plummer, J. D. 1996, ApJ, 462, 732

Hunter, D. A., \& Thronson, H. A. 1996, ApJ, 461, 202

Landolt, A. U. 1992, AJ, 104, 340

Koo, B. C., \& McKee, C. F. 1992a, ApJ, 388, 93

. 1992b, ApJ, 388, 103 
Leitherer, C., Robert, C., \& Drissen, L. 1992, ApJ, 401, 596

Marconi, G., Tosi, M., Greggio, L., \& Focardi, P. 1995, AJ, 109, 173

Martimbeau, N., Carignan, C., \& Roy, J.-R. 1994, AJ, 107, 543

Massey, P., Armandroff, T., De Veny, J., Claver, C., Harmer, C., Jacoby, G., Schoening, B., \& Silva, D. 1997, Direct Imaging Manual for Kitt Peak (Tuscon: KPNO)

Massey, P., Johnson, K. E., \& De Gioia-Eastwood, K. 1995, ApJ, 454, 151

Massey, P., Lang, C. C., De Gioia-Eastwood, K., \& Garmany, C. D. 1995, ApJ, 438, 188

Minniti, D., \& Zijlstra, A. A. 1996, ApJ, 467, L13

Noguchi, M. 1987, MNRAS, 228, 635

Odewahn, S. C. 1991, AJ, 101, 8290

Ohta, K., Tomita, A., Saito, M., Sasaki, M., \& Nakai, N. 1993, PASJ, 45, L21

Olsen, K. A. G., Hodge, P. W., Wilcots, E. M., \& Pastwick, L. 1997, ApJ, 475,545

Phillips, A. C. 1996, in ASP Conf. Ser. 91, Barred Galaxies, ed. R. Buta, D. A. Crocker, \& B. G. Elmegreen (San Francisco: ASP), 44

Piotto, G., Capaccioli, M., \& Pellegrini, C. 1994, A\&A, 287, 371

Points, S. D., Chu, Y.-H., Kim, S., Smith, R. C., Snowden, S. L., Brandner, W., \& Gruendl, R. A. 1998, ApJ, in press

Puche, D., \& Westpfahl, D. 1994, in Dwarf Galaxies, ed. G. Meylan \& P. Prugniel (Garching: ESO), 273

Puche, D., Westpfahl, D., Brinks, E., \& Roy, J.-R. 1992, AJ, 103, 1841

Ratnatunga, K. U., \& Bahcall, J. N. 1985, ApJS, 59, 63

Reid, N., Mould, J., \& Thompson, I. 1987, ApJ, 323, 433

Rhode, K. L., Salzer, J. J., \& Westpfahl, D. J. 1997, BAAS, 29, 1342
Saio, H., Kato, M., \& Nomoto, K. 1988, ApJ, 331, 388

Saito, M., Sasaki, M., Ohta, K., \& Yamada, T. 1992, PASJ, 44, 593

Sakai, S., Madore, B. F., \& Freedman, W. L. 1996, ApJ, 461, 713 (SMF)

Sandage, A., \& Carlson, G. 1982, ApJ, 258, 439 (SC82) 1985, AJ, 90, 1019 (SC85)

Schwarz, M. P. 1984, MNRAS, 209, 93

Skillman, E. D., Bomans, D. J., \& Kobulnicky, H. A. 1997, ApJ, 474, 205

Skillman, E. D., Kennicutt, R. C., \& Hodge, P. W. 1989, ApJ, 347, 875

Skillman, E. D., Terlevich, R., Teuben, P. J., \& van Woerden, H. 1988, A\&A, 198, 33

Stetson, P. B. 1987, PASP, 99, 191 1990, PASP, 102, 932

Stetson, P. B., Davis, L. E., \& Crabtree, D. R. 1990, in ASP Conf. Ser. 8, CCDs in Astronomy, ed. G. H. Jacoby (San Francisco: ASP), 289

Tenorio-Tagle, G., \& Bodenheimer, P. 1988, AR\&A, 26, 145

Tongue, T. D., \& Westpfahl, D. J. 1995, AJ, 109, 2462

Tosi, M., Greggio, L., Marconi, G., \& Focardi, P. 1991, AJ, 102, 951

Tully, R. B. 1988, Nearby Galaxies Catalog (Cambridge: Cambridge Univ. Press)

van Zee, L., Haynes, M. P., \& Giovanelli, R. 1995, AJ, 109, 990

Wade, R. A., Hoessel, J. G., Elias, J. H., \& Huchra, J. P. 1979, PASP, 91, 35

Walker, A. R. 1987, MNRAS, 224, 935 (W87)

Waller, W. 1990, PASP, 102, 1217

Westpfahl, D. J., \& Puche, D. 1994, preprint

Wilcots, E. M., Lehman, C., \& Miller, B. 1996, AJ, 111, 1575

Woosley, S. E. 1988, ApJ, 330, 218 